\title{
Consideraciones metodológicas para evaluar la calidad de vida
}

\author{
Elizabeth Velarde-Jurado MC, ${ }^{(1)}$ C arlos Avila-Figueroa MC, DC .11
}

\begin{abstract}
Velarde-Jurado E,Avila-Figueroa C. Consideraciones metodológicas para evaluar la calidad de vida. Salud Publica Mex 2002;44:448-463. El texto completo en inglés de este artículo está disponible en: http://www.insp.mx/salud/index.html
\end{abstract}

\section{Resumen}

Este trabajo hace una revisión sobre los aspectos metodológicos necesarios para evaluar la validez y consistencia de las escalas de medición de calidad de vida. La evaluación de la calidad de vida es sumamente importante, pero difícil de cuantificar objetivamente. La calidad de vida es un fenómeno que se afecta tanto por la enfermedad como por los efectos adversos del tratamiento. Las mediciones pueden estar basadas en encuestas directas a los pacientes, con referencia al inicio de la enfermedad, su diagnóstico y a los cambios de síntomas a través del tiempo. Debido a que la calidad de vida se basa en mediciones blandas con una carga variable de subjetividad, se requiere de métodos de evaluación válidos, reproducibles y confiables. Actualmente contamos con métodos objetivos que mediante cuestionarios generan escalas e índices que permiten medir las dimensiones que conforman el estado de salud. Los instrumentos para medir calidad de vida deben verse como herramientas adicionales del clínico en la evaluación integral del paciente, y en la conducción de ensayos clínicos. Los instrumentos para medir calidad de vida se clasifican en instrumentos genéricos y específicos. Los primeros son útiles para comparar diferentes poblaciones y padecimientos, pero tienen el riesgo de ser poco sensibles a los cambios clínicos, por lo cual su finalidad es meramente descriptiva. Los instrumentos específicos se basan en las características especiales de un determinado padecimiento, sobre todo para evaluar cambios físicos y efectos del tratamiento a través del tiempo. Estos nos dan mayor capacidad de discriminación

\author{
Velarde-Jurado E,Avila-Figueroa C. \\ Methods for quality \\ of life assessment. \\ Salud Publica Mex 2002;44:448-463. \\ The English version of this paper \\ is available at: http://www.insp.mx/salud/index.html
}

\begin{abstract}
A bstract
This paper reviews the methods and principles for quality of life assessment. The aging of the population and the improved survival of people with acute and chronic conditions have produced several levels of disability requiring long-term treatment and rehabilitation. In 1948 the W orld Health 0 rganization defined health as not merely the absence of disease but rather a state of complete physical, mental, and social well being. This term evolved from its conceptual definition to the development of scales to measure the quality of life beyond physical status. Thus, quality of life assessment includes areas such as mental health, social support, and life satisfaction. It is recognized that the expectations, vitality, pain, disability, and personal experiences influence the perception of a person's general health. A composite measurement aimed to quantify health according to physical, mental, and social well being simultaneously would likely include people at clearly different points on the three different continua, but in the midranges of the composite. The multidimensionality problems and the level of subjectivity involved in the assessment of the quality of life require valid and reliable instruments. This paper present an inventory of 126 questionnaires aimed to measure the quality of life for several diseases and populations. A better understanding of the methods to assess the quality of life will allow the incorporation of these instruments in the comprehensive assessment of patients, into clinical trials, and for health services research. The English version of this paperis available at: http://www.insp.mx/salud/index.html
\end{abstract}

(1) Hospital Infantil de México "Federico Gómez", México, D.F., México

Fecha de recibido: $\mathbf{8}$ de mayo de $\mathbf{2 0 0 1} \bullet$ Fecha de aprobado: $\mathbf{2 2}$ de enero de $\mathbf{2 0 0 2}$

Solicitud de Sobretiros: Dr. Carlos Avila-Figueroa. D epartamento de Epidemiología, Hospital Infantil de México "Federico Gómez". Doctor Márquez 162, Delegación Cuauhtémoc, 06720, México, D. F., México.

Correo electrónico: carlosavilaf@usa.net 
y predicción, y son particularmente útiles para ensayos clínicos. Finalmente, se presenta un inventario de los instrumentos genéricos y específicos disponibles para evaluar la calidad de vida. A demás se analizan los instrumentos en relación con su objetivo, dimensiones y función. El texto completo en inglés de este artículo está disponible en: http:// www.insp.mx/salud/index.html

Palabras clave: calidad de vida; validez; reproducibilidad de resultados; confiabilidad; nivel de salud; México
Key words: quality of life; validity; reliability; reproducibility; health assessment; Mexico
E 1 concepto de calidad de vida ha venido cobrando importancia ya que la mayor sobrevida de la población ha mejorado a expensas de un mayor número de personas con algún grado de discapacidad, y de personas con enfermedades crónicas que padecen los efectos de su enfermedad y del mismo tratamiento. Debido a que la calidad de vida se basa en mediciones blandas con una carga variable de subjetividad, se requiere de métodos de evaluación válidos, reproducibles y confiables. El mejor conocimiento de las evaluaciones para medir calidad de vida permitirá incorporar estos instrumentos en la evaluación integral de individuos, en la conducción de ensayos clínicos y en la investigación de servicios de salud.

Existen múltiples instrumentos que se han diseñado para evaluar las dimensiones que integran las mediciones de salud y de calidad de vida. Se deben considerar algunos conceptos básicos al evaluar calidad de vida ya que, siendo un concepto multidimensional, es difícil decidir qué variables deben incluirse y ello depende de la finalidad del estudio. Los instrumentos para medir la calidad de vida se han diseñado con diversos propósitos. Para conocer y comparar el estado de salud entre poblaciones (aspecto fundamental para estrategias y programas en políticas de salud), y para evaluar el impacto de ciertas intervenciones terapéuticas para modificar los síntomas y función física a través del tiempo. La calidad de vida es un fenómeno que se afecta tanto por la enfermedad como por el tratamiento (por sus efectos adversos). Los instrumentos para medir calidad de vida deben verse como herramientas adicionales del clínico en la evaluación integral del paciente y en la conducción de ensayos clínicos. ${ }^{1}$

El objetivo del presente trabajo es hacer una revisión de los aspectos metodológicos en los que se basa la evaluación de la calidad de vida, así como de proporcionar un inventario de los instrumentos genéricos y específicos disponibles para evaluarla.

\section{Evaluación de instrumentos}

Los lineamientos que sirven de referencia para el diseño de un instrumento han sido revisados cuidadosamente por Bombardier y Tugwell. ${ }^{2}$

Objetivo. Se debe evaluar claramente el objetivo del instrumento: ¿qué enfermedad se va estudiar?, y ¿en qué población se va aplicar? Aunque es muy atractivo elaborar un instrumento nuevo, es importante recordar que la elaboración y validación consumen mucho tiempo y no se tiene la certeza de que será útil. Es por ello que se recomienda usar instrumentos ya existentes; muchos de ellos son adecuados y pueden aplicarse a los propósitos del estudio. No existe el instrumento perfecto, por lo cual debe buscarse su función y aplicación dependiendo del tipo de padecimiento, y población de enfermos.

Función. Hay que definir el instrumento en función de su capacidad de discriminación, descripción y predicción de la calidad de vida. La discriminación es la propiedad que sirve para establecer diferencias entre padecimientos o entre enfermos con una misma enfermedad, y que permite estratificar poblaciones. Idealmente, el instrumento será capaz de describir la evolución del padecimiento y detectar cambios en la calidad de vida a través del tiempo. El instrumento también tendrá la capacidad de predecir el tipo de discapacidad futura y un desenlace poco afortunado. Esta función es útil para decidir la intensidad de tratamiento.

Selección de preguntas. Se asume que los cuestionarios son la forma habitual para medir calidad de vida. Se puede recurrir a un consenso de expertos en el tema, investigadores y pacientes para definir las preguntas relevantes. Una forma de analizar la utilidad de los cuestionarios es empleando técnicas estadísticas. El análisis factorial y de componentes principales es de utilidad para reducir y caracterizar las dimensiones que 
se están midiendo. Puesto que muchas preguntas en un cuestionario pueden estar correlacionadas y tener equivalencias, el primer paso sería eliminar colinariedad, redundancia y obtener una combinación que, con el mínimo número de variables, mida la misma dimensión. El análisis factorial es un método multivariable, que se emplea para explicar las relaciones entre un número importante de variables correlacionadas e identificar unos cuantos factores independientes, que conceptualmente sean significativos. ${ }^{2}$

Contenido. Deben incluirse dimensiones importantes como el autocuidado, la actividad física, la comunicación, la interacción social, el descanso, las actividades recreativas y las repercusiones emocionales.

Formas de respuesta. Se pueden contestar de diversas formas, tomándose en cuenta que si se suman las calificaciones de las preguntas relacionadas con la actividad física se puede obtener una evaluación representativa de dicha área.

Sentido biológico. El instrumento debe comportarse de acuerdo con teorías que se vinculen con la calidad de vida. En la medida en que los instrumentos cumplan con las predicciones clínicas lograrán mayor credibilidad. Estas mediciones representan un complemento para el cuidado integral del paciente.

Factibilidad. Este es un aspecto trascendente; estos instrumentos tienen que ser adecuados para las diferentes culturas en las cuales se aplican. Se debe buscar que las preguntas se entiendan y se acepten para poderse contestar. Debe recalcarse que el tiempo que consume su aplicación puede limitarla en la práctica clínica. ${ }^{3,4}$

\section{Validez y consistencia}

Es la aproximación que se hace al valor real o "verdadero" de la característica que se quiere medir; más sencillamente, "medir lo que realmente se quiere medir". Una característica que debe tener una medición es la consistencia; los datos obtenidos tendrán calidad científica, si durante el proceso de medición pueden ser consistentemente repetibles por el mismo observador $\mathrm{u}$ otros. ${ }^{5}$ La medición será científicamente aceptada cuando tenga consistencia y pueda ser reproducible. Feinstein ${ }^{6}$ recomienda usar el término consistencia en el desarrollo de un índice de medición del estado de salud del individuo. Si se tiene consistencia en una medición, la exactitud se podrá obtener estableciendo un estándar de referencia. Este último se obtendrá mediante la utilización de un procedimiento que produzca mediciones objetivas, dimensiones de una entidad, o puede ser la opinión de consenso de un grupo de expertos. Las mediciones de la consistencia, conocidas como duras $u$ objetivas, tienen varias características: a) exactitud, es el procedimiento para obtener una medición que se acerque lo mejor posible a la medición "real" y comparable con un índice o una medición previamente estandarizada; b) objetividad, se refiere al hecho de que una medición esté libre de juicios humanos; c) dimensionalidad, implica que una medición debe estar referida en escalas estandarizadas de valores numéricos; $d$ ) preservabilidad, se refiere a que la medición puede ser reexaminada posteriormente. Los problemas metodológicos que enfrenta la medición de calidad de vida son dos; primero, que se trata de medidas consideradas "blandas", y segundo, que no hay un valor de referencia o estándar de oro contra el cual se pueda comparar. ${ }^{3,5,7}$

Validez. Esta se realiza para demostrar la utilidad clínica de un cuestionario, de tal forma que los datos obtenidos sean confiables. Para que una medición se considere con validez debe constar con tres características: a) consistencia, se refiere al hecho de conocer si el índice de medición tiene consistencia, la cual es un proceso intrínseco del proceso de validación de cualquier índice de medición; b) exactitud, se refiere al hecho de comparar el índice o la medición realizada con un índice previamente estandarizado; y c) adecuada, que el índice sea satisfactorio y adecuado para el fin que fue creado. Hay varias formas y conceptos de validez que se definen como sigue:

Validez aparente. Es una evaluación cualitativa, se conocen los componentes y el aspecto grueso del índice Validez de contenido. Es una valoración cualitativa que se refiere a la evaluación integral que hace el índice del atributo clínico que desea medir

Validez de criterio. Este tipo de validez se lleva a cabo cuando se tiene un índice estandarizado o "estándar de oro", contra el cual se compara un nuevo índice desarrollado; cuando se tiene índice estandarizado se calculan sensibilidad, especificidad y los valores predictivos positivos y negativos del nuevo índice Validez de constructo. Aspecto que se evalúa con un juicio cualitativo; se observa si existe relación entre las variables tomadas por el índice y lo que éste quiere evaluar. Para validez de constructo se aplica el índice, se observan los resultados y se corrobora si evalúa lo que queremos conocer. $3,5,7,8$

Consistencia interna. Con el propósito de validar un instrumento se debe demostrar que es consistente o confiable. La consistencia de una escala de medición compuesta por preguntas múltiples debe ser demostrada, ya que no existe manera de compararla con una variable externa. La consistencia interna tiene dos componentes: 
La consistencia de ejecución. Se refiere a la respuesta individual de las preguntas que tiene el índice desarrollado, o la repetición del índice por el mismo usuario. Mide la variabilidad del contenido de las preguntas de un índice y señala su consistencia. Se evalúa mediante la consistencia de la mitad de la prueba, si se obtiene la misma puntuación con la aplicación completa de la prueba y con la aplicación de la mitad de la prueba. En consistencia (Test-retest) se aplica el cuestionario y se repite para evaluar su correlación en diferentes tiempos.

Consistencia de homogeneidad. Evalúa la consistencia total de un índice (dominio); se refiere a la consistencia que se da en las relaciones que tienen las diferentes preguntas (ítems) utilizadas en la construcción de un índice general, con el que se desea conocer determinado atributo. Se utiliza cuando se evalúan variables subjetivas conocidas también como medidas blandas. Puede estar constituido por la suma de una serie de preguntas individuales. ${ }^{9}$ Para conocer qué tan consistentemente las preguntas que se han incluido miden lo que se quiere medir, se evalúan las interrelaciones de estas variables entre sí. Se refiere a la coherencia de las preguntas y a la interrelación de ellas en la medición de determinado atributo. Para evaluar la homogeneidad interna de una escala que contiene un grupo de preguntas llamada batería, se calcula el coeficiente "alfa de Cronbach". ${ }^{10}$ El coeficiente de alfa evalúa la confiabilidad de una escala nominal. Si la presentación de las preguntas es binaria, deberá calcularse el coeficiente de correlación Kuder Richardson. ${ }^{5}$ Estos coeficientes representan un promedio ponderado de las interrelaciones que existen entre las preguntas de un índice, su valor se encontrará entre 0.1 y 1.0 ; se considera un buen índice cuando el valor del mismo es de 0.80 y excelente si es superior a $0.90 .^{5}$

La consistencia externa. Se refiere a la variabilidad externa de la medición cuando se aplica en diferentes ocasiones por el mismo o por diferentes observadores. Es decir, si la medición es consistente al aplicarla externamente, y se obtienen los mismos resultados a pesar de que se realicen mediciones en más de una ocasión. Esta se alcanza cuando se mide un fenómeno o característica clínica mediante: a) observación del fenómeno; b) las observaciones se concentran en un grupo de datos, y c) los datos se convierten en una escala para medir el fenómeno. En la fase a y b deben incluirse especificaciones operacionales para convertir el grupo de datos observados en variables particulares, categorías y otros aspectos para la construcción de un índice de medición. A este proceso se le conoce como "elementos de criterio". Para la evaluación de la consistencia externa pueden realizarse cálculos in- directos de la misma, pudiendo utilizar coeficientes de concordancia y coeficientes de correlación.

Pueden utilizarse los coeficientes de correlación de Pearson, de Spearman o de Tau de Kendal. Estos evalúan tendencia y no concordancia, por lo que no controlan el sesgo sistemático si estuviera presente en los datos..$^{11}$ Los coeficientes de concordancia toman en cuenta la concordancia que pudiera existir por el azar. Miden concordancia y no la tendencia. Cuando la variable se mide en escala binaria o nominal, y sólo son dos observaciones u observadores, se recomienda utilizar el coeficiente kappa no ponderado (K). Una vez que se tiene el valor de (K), éste se interpreta siguiendo los lineamientos de Landis y Koch. ${ }^{12}$ Cuando la variable se mide en escala ordinal, y son sólo dos observaciones $\mathrm{u}$ observadores, se recomienda utilizar el coeficiente kappa ponderado (Kp). Se acepta que un $(\mathrm{Kp})$ es una concordancia aceptable cuando se tiene un valor entre 0.5 y 0.6 . Para conocer la concordancia de dos o más observadores cuando la variable se mide en escala continua, se recomienda la utilización del coeficiente de correlación de intraclase. Este se basa en un análisis de varianza de la variabilidad de las mediciones, tanto de los observadores que miden como la de los residuales. Puede usarse la formula de Brako, la cual acepta como buen índice de concordancia un valor mínimo de $0.75 .5,13$

\section{Instrumentos disponibles para medir calidad de vida}

Los instrumentos para medir calidad de vida se clasifican en instrumentos genéricos y específicos. Los primeros son útiles para comparar diferentes poblaciones y padecimientos, pero tienen el riesgo de ser poco sensibles a los cambios clínicos, por lo cual su finalidad es meramente descriptiva. Los instrumentos específicos se basan en las características especiales de un determinado padecimiento, sobre todo para evaluar cambios físicos y efectos del tratamiento a través del tiempo. Estos nos dan mayor capacidad de discriminación y predicción, y son particularmente útiles para ensayos clínicos. ${ }^{3}$ Con el propósito de tener un panorama de los instrumentos para medir calidad de vida y que podrían ser aplicados en diferentes edades y poblaciones, en los cuadros I y II, A y B se presenta un análisis de los instrumentos publicados que cuentan con información explícita de los instrumentos en relación con su objetivo, dimensiones y función. El objetivo de la publicación contempla la evaluación de su validez y confiabilidad. También se consideró su validación en otro idioma diferente al original y cuando el propósito del estudio era hacer descripción de la cali- 
Cuadro IA

\section{Cuestionarios genéricos para eValuar la CaLidad de Vida en adultos}

\begin{tabular}{|c|c|c|c|c|c|c|}
\hline Instrumento & Objetivo & Validez & Confiabilidad & Dimensión & Función & Referenci \\
\hline $\begin{array}{l}\text { The Sickness Impact } \\
\text { Profile: SIP }\end{array}$ & $\begin{array}{l}\text { Validación } \\
\text { Confiabilidad }\end{array}$ & $\begin{array}{l}\text { Contenido } \\
\text { Constructo }\end{array}$ & $\begin{array}{l}\text { Prueba repetida } \\
\text { Consistencia interna }\end{array}$ & $\begin{array}{l}\text { Movilidad físico, } \\
\text { emocional, social }\end{array}$ & $\begin{array}{l}\text { Predicción } \\
\text { Evaluación }\end{array}$ & 27 \\
\hline $\begin{array}{l}\text { The Sickness Impact } \\
\text { Profile: SIP }\end{array}$ & $\begin{array}{l}\text { Validación } \\
\text { Confiabilidad } \\
\text { Traducción al español }\end{array}$ & $\begin{array}{l}\text { Contenido } \\
\text { Constructo }\end{array}$ & $\begin{array}{l}\text { Prueba repetida } \\
\text { Consistencia interna }\end{array}$ & $\begin{array}{l}\text { Funcionalidad físico, } \\
\text { emocional, social }\end{array}$ & $\begin{array}{l}\text { Discriminación } \\
\text { Evaluación }\end{array}$ & 28 \\
\hline $\begin{array}{l}\text { The Sickness Impact } \\
\text { Profile: SIP }\end{array}$ & $\begin{array}{l}\text { Validación } \\
\text { Confiabilidad } \\
\text { Traducción al español }\end{array}$ & $\begin{array}{l}\text { Contenido } \\
\text { Constructo }\end{array}$ & $\begin{array}{l}\text { Prueba repetida } \\
\text { Consistencia interna }\end{array}$ & $\begin{array}{l}\text { Funcionalidad físico, } \\
\text { emocional, social }\end{array}$ & $\begin{array}{l}\text { Discriminación } \\
\text { Evaluación }\end{array}$ & 29 \\
\hline The Dartmouth - COOP & $\begin{array}{l}\text { Validación } \\
\text { Confiabilidad }\end{array}$ & $\begin{array}{l}\text { Constructo } \\
\text { Contenido } \\
\text { Criterio }\end{array}$ & $\begin{array}{l}\text { Prueba repetida } \\
\text { Consistencia interna }\end{array}$ & $\begin{array}{l}\text { Movilidad físico, social, } \\
\text { emocional, apoyo } \\
\text { social, dolor }\end{array}$ & $\begin{array}{l}\text { Discriminación } \\
\text { Evaluación }\end{array}$ & 30 \\
\hline $\begin{array}{l}\text { The McMaster Health } \\
\text { Index Q uestionnaire }\end{array}$ & $\begin{array}{l}\text { Validación } \\
\text { Confiabilidad }\end{array}$ & $\begin{array}{l}\text { Contenido } \\
\text { Constructo }\end{array}$ & Prueba repetida & $\begin{array}{l}\text { Movilidad físico, } \\
\text { emocional, social }\end{array}$ & $\begin{array}{l}\text { Discriminación } \\
\text { Evaluación }\end{array}$ & 31 \\
\hline $\begin{array}{l}\text { Q uality of life Index: } \\
\text { Q L-Index }\end{array}$ & $\begin{array}{l}\text { Validación } \\
\text { Confiabilidad } \\
\text { Descripción }\end{array}$ & Constructo & Consistencia interna & $\begin{array}{l}\text { Funcionalidad físico, } \\
\text { emocional, social, } \\
\text { vitalidad }\end{array}$ & Discriminación & 32 \\
\hline $\begin{array}{l}\text { The N ottingham Health } \\
\text { Profile: N HP }\end{array}$ & $\begin{array}{l}\text { Validación } \\
\text { Traducción al español }\end{array}$ & Constructo & Consistencia interna & $\begin{array}{l}\text { Funcionalidad físico, } \\
\text { emocional, social, } \\
\text { nivel actividad, } \\
\text { percepción deterioro }\end{array}$ & Discriminación & 33 \\
\hline The Dartmouth - COOP & $\begin{array}{l}\text { Validación } \\
\text { Confiabilidad } \\
\text { Traducción al español }\end{array}$ & $\begin{array}{l}\text { Constructo } \\
\text { Contenido } \\
\text { Criterio }\end{array}$ & $\begin{array}{l}\text { Prueba repetida } \\
\text { Consistencia interna }\end{array}$ & $\begin{array}{l}\text { Movilidad físico, social, } \\
\text { emocional, apoyo } \\
\text { social, dolor }\end{array}$ & $\begin{array}{l}\text { Discriminación } \\
\text { Evaluación }\end{array}$ & 22 \\
\hline $\begin{array}{l}\text { The Duke Health Profile: } \\
\text { DUKE }\end{array}$ & $\begin{array}{l}\text { Validación } \\
\text { Confiabilidad }\end{array}$ & $\begin{array}{l}\text { Constructo } \\
\text { Criterio }\end{array}$ & $\begin{array}{l}\text { Prueba repetida } \\
\text { Consistencia interna }\end{array}$ & $\begin{array}{l}\text { Funcionalidad físico, } \\
\text { social, emocional, } \\
\text { auto estima, dolor, } \\
\text { apoyo social }\end{array}$ & $\begin{array}{l}\text { Discriminación } \\
\text { Predicción }\end{array}$ & 34 \\
\hline $\begin{array}{l}\text { The Functional Status } \\
\text { Index: FSI }\end{array}$ & $\begin{array}{l}\text { Validación } \\
\text { Confiabilidad }\end{array}$ & $\begin{array}{l}\text { Constructo } \\
\text { Contenido }\end{array}$ & Consistencia interna & $\begin{array}{l}\text { Funcionalidad, físico, } \\
\text { social emocional }\end{array}$ & $\begin{array}{l}\text { Discriminación } \\
\text { Evaluación }\end{array}$ & 35 \\
\hline $\begin{array}{l}\text { The Kamofsky } \\
\text { Performance Status (KPS) } \\
\text { Scale }\end{array}$ & $\begin{array}{l}\text { Validación } \\
\text { Confiabilidad } \\
\text { Descripción }\end{array}$ & Constructo & $\begin{array}{l}\text { Prueba repetida } \\
\text { Consistencia interna }\end{array}$ & Funcionalidad, dolor & $\begin{array}{l}\text { Discriminación } \\
\text { Predicción }\end{array}$ & 36 \\
\hline $\begin{array}{l}\text { The general Health } \\
\text { Q uestionnaire: GHQ -28 }\end{array}$ & $\begin{array}{l}\text { Validación } \\
\text { Confiabilidad } \\
\text { Descripción }\end{array}$ & Constructo & $\begin{array}{l}\text { Prueba repetida } \\
\text { Consistencia interna }\end{array}$ & $\begin{array}{l}\text { Funcionalidad, social, } \\
\text { emocional, ansiedad }\end{array}$ & Discriminación & 37 \\
\hline $\begin{array}{l}\text { The Health Assessment } \\
\text { Q uestionnaire: HAQ }\end{array}$ & $\begin{array}{l}\text { Validación } \\
\text { Confiabilidad } \\
\text { Descripción }\end{array}$ & Constructo & $\begin{array}{l}\text { Prueba repetida } \\
\text { Consistencia interna }\end{array}$ & $\begin{array}{l}\text { Funcionalidad físico, } \\
\text { social, mental, dolor, } \\
\text { autocuidado }\end{array}$ & $\begin{array}{l}\text { Discriminación } \\
\text { Predicción }\end{array}$ & 38 \\
\hline $\begin{array}{l}\text { The Sickness Impact } \\
\text { Profile: SIP } 68 \text { Short } \\
\text { Generic Version }\end{array}$ & $\begin{array}{l}\text { Validación } \\
\text { Confiabilidad }\end{array}$ & Constructo & $\begin{array}{l}\text { Prueba repetida } \\
\text { Consistencia interna }\end{array}$ & $\begin{array}{l}\text { Funcionalidad, físico, } \\
\text { emocional, social, } \\
\text { autonomía, satisfacción }\end{array}$ & $\begin{array}{l}\text { Discriminación } \\
\text { Predicción } \\
\text { Evaluación }\end{array}$ & 39 \\
\hline $\begin{array}{l}\text { The Q uality of W ell-Being } \\
\text { Scale: Q W B }\end{array}$ & $\begin{array}{l}\text { Validación } \\
\text { Confiabilidad } \\
\text { Descripción }\end{array}$ & $\begin{array}{l}\text { Contenido } \\
\text { Constructo }\end{array}$ & Consistencia interna & $\begin{array}{l}\text { Funcionalidad físico, } \\
\text { emocional, social, nivel } \\
\text { actividad, deterioro }\end{array}$ & $\begin{array}{l}\text { Discriminación } \\
\text { Predicción }\end{array}$ & 40 \\
\hline $\begin{array}{l}\text { The MOS-Short-form } \\
\text { General Health Survey: }\end{array}$ & $\begin{array}{l}\text { Validación } \\
\text { Confiabilidad }\end{array}$ & $\begin{array}{l}\text { Constructo } \\
\text { Criterio }\end{array}$ & $\begin{array}{l}\text { Prueba repetida } \\
\text { Consistencia interna }\end{array}$ & $\begin{array}{l}\text { Funcionalidad físico, } \\
\text { social, emocional, dolor, } \\
\text { vitalidad }\end{array}$ & Discriminación & 41 \\
\hline $\begin{array}{l}\text { The Medical O utcomes } \\
\text { Study Short-form Health } \\
\text { Survey: MOS }\end{array}$ & $\begin{array}{l}\text { Validación } \\
\text { Confiabilidad } \\
\text { Descripción }\end{array}$ & Constructo & $\begin{array}{l}\text { Prueba repetida } \\
\text { Consistencia interna }\end{array}$ & $\begin{array}{l}\text { Funcionalidad físico, } \\
\text { social, emocional, dolor }\end{array}$ & Discriminación & 42 \\
\hline
\end{tabular}

\section{(Continúa)}




\section{Cuadro IA \\ (Continuación)}

\begin{tabular}{|c|c|c|c|c|c|c|}
\hline $\begin{array}{l}\text { Cuestionario Criterio de } \\
\text { Calidad deVida: CCV }\end{array}$ & $\begin{array}{l}\text { Validación } \\
\text { Confiabilidad }\end{array}$ & Constructo & Consistencia interna & $\begin{array}{l}\text { Funcionalidad físico, } \\
\text { social, emocional, } \\
\text { vómito, apetito, sueño, } \\
\text { fatiga, dolor }\end{array}$ & Discriminación & 43 \\
\hline $\begin{array}{l}\text { The McGill Pain } \\
\text { Q uestionnaire: MQ O L }\end{array}$ & $\begin{array}{l}\text { Validación } \\
\text { Confiabilidad } \\
\text { Descripción }\end{array}$ & $\begin{array}{l}\text { Constructo } \\
\text { Criterio }\end{array}$ & $\begin{array}{l}\text { Prueba repetida } \\
\text { Consistencia interna }\end{array}$ & $\begin{array}{l}\text { Funcionalidad físico, } \\
\text { social, emocional, } \\
\text { espiritual, apoyo social }\end{array}$ & Discriminación & 44 \\
\hline $\begin{array}{l}\text { The European Research } \\
\text { and Treatment Q uality Life- } \\
\text { Q uestionnaire: EO RTC } \\
\text { Q LQ -C } 36\end{array}$ & $\begin{array}{l}\text { Validación } \\
\text { Confiabilidad }\end{array}$ & Criterio & $\begin{array}{l}\text { Prueba repetida } \\
\text { Consistencia interna }\end{array}$ & $\begin{array}{l}\text { Funcionalidad físico, } \\
\text { social, emocional, } \\
\text { fatiga, dolor }\end{array}$ & Discriminación & 45 \\
\hline $\begin{array}{l}\text { The European Research and } \\
\text { Treatment Q uality Life- } \\
\text { Q uestionnaire: EO RTC } \\
\text { Q LQ -C } 30\end{array}$ & $\begin{array}{l}\text { Validación } \\
\text { Confiabilidad }\end{array}$ & Criterio & $\begin{array}{l}\text { Prueba repetida } \\
\text { Consistencia interna }\end{array}$ & $\begin{array}{l}\text { Funcionalidad físico, } \\
\text { social, emocional, } \\
\text { fatiga, dolor }\end{array}$ & $\begin{array}{l}\text { Discriminación } \\
\text { Evaluación }\end{array}$ & 46 \\
\hline $\begin{array}{l}\text { The General Health } \\
\text { Perception: GHP }\end{array}$ & $\begin{array}{l}\text { Validación } \\
\text { Confiabilidad }\end{array}$ & Criterio & $\begin{array}{l}\text { Prueba repetida } \\
\text { Consistencia interna }\end{array}$ & $\begin{array}{l}\text { Funcionalidad físico, } \\
\text { social, emocional, } \\
\text { vitalidad, mental, dolor }\end{array}$ & $\begin{array}{l}\text { Discriminación } \\
\text { Predicción }\end{array}$ & 47 \\
\hline $\begin{array}{l}\text { The W isconsin } Q \text { uality of } \\
\text { Life Index-C anadian } \\
\text { version: } C a W-Q \text { LI }\end{array}$ & $\begin{array}{l}\text { Validación } \\
\text { Confiabilidad } \\
\text { Traducción al inglés } \\
\text { y francés }\end{array}$ & $\begin{array}{l}\text { Constructo } \\
\text { Criterio }\end{array}$ & $\begin{array}{l}\text { Prueba repetida } \\
\text { Consistencia interna }\end{array}$ & $\begin{array}{l}\text { Funcionalidad físico, } \\
\text { social, emocional, } \\
\text { satisfacción, apoyo } \\
\text { social }\end{array}$ & $\begin{array}{l}\text { Discriminación } \\
\text { Predicción }\end{array}$ & 48 \\
\hline $\begin{array}{l}\text { The European Research } \\
\text { Q uestionnaire Q uality of } \\
\text { Life: EURO Q OL 5D }\end{array}$ & $\begin{array}{l}\text { Validación } \\
\text { Confiabilidad }\end{array}$ & Criterio & Consistencia interna & $\begin{array}{l}\text { Funcionalidad físico, } \\
\text { autocuidado, ansiedad, } \\
\text { limitación }\end{array}$ & Discriminación & 49 \\
\hline Encuesta: SF-36 & $\begin{array}{l}\text { Validación } \\
\text { Confiabilidad }\end{array}$ & $\begin{array}{l}\text { Constructo } \\
\text { Criterio }\end{array}$ & $\begin{array}{l}\text { Prueba repetida } \\
\text { Consistencia interna }\end{array}$ & $\begin{array}{l}\text { Funcionalidad físico, } \\
\text { social, emocional, } \\
\text { dolor }\end{array}$ & $\begin{array}{l}\text { Discriminación } \\
\text { Evaluación }\end{array}$ & 26 \\
\hline $\begin{array}{l}\text { The Modified Health } \\
\text { Assessment Q uestionnaire: } \\
\text { M-HAQ }\end{array}$ & $\begin{array}{l}\text { Validación } \\
\text { Confiabilidad }\end{array}$ & $\begin{array}{l}\text { Constructo } \\
\text { Criterio }\end{array}$ & Consistencia interna & $\begin{array}{l}\text { Funcionalidad físico, } \\
\text { social, emocional, } \\
\text { ansiedad }\end{array}$ & $\begin{array}{l}\text { Discriminación } \\
\text { Predicción }\end{array}$ & 50 \\
\hline $\begin{array}{l}\text { The Multidimensional Index } \\
\text { of Q uality of Life: MIQ L }\end{array}$ & $\begin{array}{l}\text { Validación } \\
\text { Confiabilidad }\end{array}$ & Constructo & Consistencia interna & $\begin{array}{l}\text { Funcionalidad físico, } \\
\text { social, emocional, } \\
\text { cognición }\end{array}$ & Discriminación & 51 \\
\hline $\begin{array}{l}\text { The RAND } 36 \text {-Item Health } \\
\text { Survey Q uestionnaire: } \\
\text { RAND-SF-36 }\end{array}$ & $\begin{array}{l}\text { Validación } \\
\text { Confiabilidad }\end{array}$ & Constructo & Consistencia interna & $\begin{array}{l}\text { Funcionalidad físico, } \\
\text { social, emocional, dolor, } \\
\text { energía, fatiga }\end{array}$ & $\begin{array}{l}\text { Discriminación } \\
\text { Predicción }\end{array}$ & 52 \\
\hline $\begin{array}{l}\text { The Perceived Q uality of } \\
\text { Life Scale: PQ oL }\end{array}$ & $\begin{array}{l}\text { Validación } \\
\text { Confiabilidad }\end{array}$ & Constructo & Consistencia interna & $\begin{array}{l}\text { Funcionalidad físico, } \\
\text { social, emocional, dolor, } \\
\text { energía, fatiga, depresión }\end{array}$ & $\begin{array}{l}\text { Discriminación } \\
\text { Predicción }\end{array}$ & 53 \\
\hline $\begin{array}{l}\text { The World Health } \\
\text { O rganization Q uality of } \\
\text { Life:W HOQOL Instrument }\end{array}$ & $\begin{array}{l}\text { Validación } \\
\text { Confiabilidad }\end{array}$ & $\begin{array}{l}\text { Constructo } \\
\text { Criterio }\end{array}$ & $\begin{array}{l}\text { Prueba repetida } \\
\text { Consistencia interna }\end{array}$ & $\begin{array}{l}\text { Funcionalidad físico, } \\
\text { social, emocional, } \\
\text { autonomía }\end{array}$ & Discriminación & 54 \\
\hline $\begin{array}{l}\text { The Index of W ell-Being: } \\
\text { IW B }\end{array}$ & $\begin{array}{l}\text { Validación } \\
\text { Confiabilidad }\end{array}$ & $\begin{array}{l}\text { Constructo } \\
\text { Contenido }\end{array}$ & Prueba repetida & $\begin{array}{l}\text { Funcionalidad físico, } \\
\text { social, emocional }\end{array}$ & $\begin{array}{l}\text { Discriminación } \\
\text { Evaluación }\end{array}$ & 55 \\
\hline $\begin{array}{l}\text { The W orld Health } \\
\text { O rganization Q uality of } \\
\text { Life:W HOQ OL - } 100\end{array}$ & $\begin{array}{l}\text { Validación } \\
\text { Confiabilidad }\end{array}$ & $\begin{array}{l}\text { Constructo } \\
\text { Criterio }\end{array}$ & $\begin{array}{l}\text { Prueba repetida } \\
\text { Consistencia interna }\end{array}$ & $\begin{array}{l}\text { Funcionalidad físico, } \\
\text { emocional, social, entorno, } \\
\text { valores, autonomía }\end{array}$ & $\begin{array}{l}\text { Discriminación } \\
\text { Predicción } \\
\text { Evaluación }\end{array}$ & 56 \\
\hline $\begin{array}{l}\text { The Health and Activities } \\
\text { Limitations Index: HALEX }\end{array}$ & $\begin{array}{l}\text { Validación } \\
\text { Confiabilidad }\end{array}$ & Criterio & $\begin{array}{l}\text { Prueba repetida } \\
\text { Consistencia interna }\end{array}$ & $\begin{array}{l}\text { Funcionalidad físico, } \\
\text { social, emocional }\end{array}$ & Discriminación & 57 \\
\hline $\begin{array}{l}\text { The Medical O utcomes } \\
\text { Study Short-form Health } \\
\text { Survey: MOS 6A }\end{array}$ & $\begin{array}{l}\text { Validación } \\
\text { Confiabilidad }\end{array}$ & $\begin{array}{l}\text { Constructo } \\
\text { Criterio }\end{array}$ & $\begin{array}{l}\text { Prueba repetida } \\
\text { Consistencia interna }\end{array}$ & $\begin{array}{l}\text { Funcionalidad físico, } \\
\text { social, emocional, dolor }\end{array}$ & $\begin{array}{l}\text { Discriminación } \\
\text { Evaluación }\end{array}$ & 58 \\
\hline The Q L-Index LASA Scales & $\begin{array}{l}\text { Validación } \\
\text { Confiabilidad }\end{array}$ & Constructo & $\begin{array}{l}\text { Prueba repetida } \\
\text { Consistencia interna }\end{array}$ & $\begin{array}{l}\text { Funcionalidad físico, } \\
\text { social, emocional, fatiga, } \\
\text { dolor }\end{array}$ & Discriminación & 59 \\
\hline
\end{tabular}


Cuadro IB

\section{Cuestionarios genéricos para eVALUAR LA CaLIDAD DE VIDA EN NIÑOS Y ADOLESCENTES}

\begin{tabular}{|c|c|c|c|c|c|c|}
\hline Instrumento & Objetivo & Validez & Confiabilidad & Dimensión & Función & Referencia \\
\hline $\begin{array}{l}\text { The Q uality of W ell-Being } \\
\text { Scale: QW B }\end{array}$ & $\begin{array}{l}\text { Validación } \\
\text { Confiabilidad } \\
\text { Descripción }\end{array}$ & $\begin{array}{l}\text { Constructo } \\
\text { Contenido }\end{array}$ & Consistencia interna & $\begin{array}{l}\text { Funcionalidad físico, } \\
\text { social, emocional, social, } \\
\text { nivel actividad, percepción } \\
\text { deterioro }\end{array}$ & $\begin{array}{l}\text { Discriminación } \\
\text { Evaluación }\end{array}$ & 40 \\
\hline $\begin{array}{l}\text { Play Performance Scale for } \\
\text { Children: PPSC }\end{array}$ & $\begin{array}{l}\text { Validación } \\
\text { Confiabilidad } \\
\text { Descripción }\end{array}$ & Constructo & Consistencia interna & $\begin{array}{l}\text { Funcionalidad social, } \\
\text { física, emocional, conducta }\end{array}$ & Discriminación & 60 \\
\hline $\begin{array}{l}\text { The Physical Health Status } \\
\text { Instrument }\end{array}$ & $\begin{array}{l}\text { Validación } \\
\text { Confiabilidad } \\
\text { Descripción }\end{array}$ & Constructo & Consistencia interna & $\begin{array}{l}\text { Actividad cotidiana, social, } \\
\text { física, emocional, conducta }\end{array}$ & Discriminación & 61 \\
\hline $\begin{array}{l}\text { The Functional Status } \\
\text { Measure of Child Health: } \\
\text { FS II (R) }\end{array}$ & $\begin{array}{l}\text { Validación } \\
\text { Confiabilidad }\end{array}$ & $\begin{array}{l}\text { Constructo } \\
\text { Criterio }\end{array}$ & $\begin{array}{l}\text { Prueba repetida } \\
\text { Consistencia interna }\end{array}$ & $\begin{array}{l}\text { Actividad cotidiana, social, } \\
\text { física, emocional, cambios, } \\
\text { conducta, dolor, limitación }\end{array}$ & Discriminación & 62 \\
\hline The Dartmouth - COOP & $\begin{array}{l}\text { Validación } \\
\text { Confiabilidad } \\
\text { Traducción al español }\end{array}$ & $\begin{array}{l}\text { Constructo } \\
\text { Contenido } \\
\text { Criterio }\end{array}$ & $\begin{array}{l}\text { Prueba repetida } \\
\text { Consistencia interna }\end{array}$ & $\begin{array}{l}\text { Movilidad físico, social, } \\
\text { emocional, apoyo social, } \\
\text { dolor }\end{array}$ & $\begin{array}{l}\text { Discriminación } \\
\text { Evaluación }\end{array}$ & 22 \\
\hline $\begin{array}{l}\text { The Childhood Health } \\
\text { Assessment Q uestionnaire: } \\
\text { CHAQ }\end{array}$ & $\begin{array}{l}\text { Validación } \\
\text { Confiabilidad } \\
\text { Traducción al portugués }\end{array}$ & Constructo & $\begin{array}{l}\text { Prueba repetida } \\
\text { Consistencia interna }\end{array}$ & $\begin{array}{l}\text { Actividad social, física, } \\
\text { emocional, dolor }\end{array}$ & $\begin{array}{l}\text { Discriminación } \\
\text { Evaluación }\end{array}$ & 63 \\
\hline $\begin{array}{l}\text { The Costa Rica Childhood } \\
\text { Health-Assessment } \\
\text { Q uestionnaire: CR - CHAQ }\end{array}$ & $\begin{array}{l}\text { Validación } \\
\text { Confiabilidad } \\
\text { Traducción al español }\end{array}$ & $\begin{array}{l}\text { Constructo } \\
\text { Criterio }\end{array}$ & Prueba repetida & $\begin{array}{l}\text { Funcionalidad físico, social, } \\
\text { emocional, higiene, social, } \\
\text { mental, dolor }\end{array}$ & $\begin{array}{l}\text { Discriminación } \\
\text { Evaluación }\end{array}$ & 64 \\
\hline $\begin{array}{l}\text { The Childhood Health } \\
\text { Assessment Q uestionnaire: } \\
\text { CHAQ }\end{array}$ & $\begin{array}{l}\text { Validación } \\
\text { Confiabilidad } \\
\text { traducción al español }\end{array}$ & $\begin{array}{l}\text { Constructo } \\
\text { Criterio }\end{array}$ & $\begin{array}{l}\text { Prueba repetida } \\
\text { Consistencia interna }\end{array}$ & $\begin{array}{l}\text { Actividad física, higiene, } \\
\text { dolor, imitación }\end{array}$ & $\begin{array}{l}\text { Discriminación } \\
\text { Evaluación }\end{array}$ & 65 \\
\hline $\begin{array}{l}\text { The Childhood Health } \\
\text { Assessment Q uestionnaire: } \\
\text { CHAQ }\end{array}$ & $\begin{array}{l}\text { Validación } \\
\text { Confiabilidad }\end{array}$ & $\begin{array}{l}\text { Constructo } \\
\text { Criterio }\end{array}$ & $\begin{array}{l}\text { Prueba repetida } \\
\text { Consistencia interna }\end{array}$ & $\begin{array}{l}\text { Actividad física, higiene, } \\
\text { dolor, imitación }\end{array}$ & $\begin{array}{l}\text { Discriminación } \\
\text { Predicción } \\
\text { Evaluación }\end{array}$ & 66 \\
\hline $\begin{array}{l}\text { The Short form Health } \\
\text { Survey Q uestionnaire: } \\
\text { SF-36 }\end{array}$ & Validación & $\begin{array}{l}\text { Constructo } \\
\text { Criterio }\end{array}$ & Consistencia interna & $\begin{array}{l}\text { Funcionalidad físico, social, } \\
\text { dolor, percepción, vitalidad }\end{array}$ & $\begin{array}{l}\text { Discriminación } \\
\text { Evaluación }\end{array}$ & 67 \\
\hline $\begin{array}{l}\text { The Pediatric Q uality of } \\
\text { Life Inventory: PedsQ L. }\end{array}$ & $\begin{array}{l}\text { Validación } \\
\text { Confiabilidad }\end{array}$ & $\begin{array}{l}\text { Constructo } \\
\text { Criterio }\end{array}$ & Consistencia interna & $\begin{array}{l}\text { Funcionalidad físico, social, } \\
\text { cognición, dolor, temor, } \\
\text { ansiedad }\end{array}$ & Discriminación & 68 \\
\hline $\begin{array}{l}\text { The Short form Measuring } \\
\text { Health-related Q uality of } \\
\text { Life: HRQ OL }\end{array}$ & $\begin{array}{l}\text { Validación } \\
\text { Confiabilidad }\end{array}$ & $\begin{array}{l}\text { Constructo } \\
\text { Contenido } \\
\text { Criterio }\end{array}$ & $\begin{array}{l}\text { Prueba repetida } \\
\text { Consistencia interna }\end{array}$ & $\begin{array}{l}\text { Funcionalidad físico, social, } \\
\text { mental }\end{array}$ & $\begin{array}{l}\text { Discriminación } \\
\text { Predicción } \\
\text { Evaluación }\end{array}$ & 69 \\
\hline $\begin{array}{l}\text { The Childhood Health } \\
\text { Assessment Q uestionnaire } \\
\text { disability index: CHAQ -DI }\end{array}$ & $\begin{array}{l}\text { Validación } \\
\text { Confiabilidad } \\
\text { Traducción al español }\end{array}$ & $\begin{array}{l}\text { Constructo } \\
\text { Criterio }\end{array}$ & $\begin{array}{l}\text { Prueba repetida } \\
\text { Consistencia interna }\end{array}$ & $\begin{array}{l}\text { Funcionalidad físico, social, } \\
\text { emocional, higiene, dolor, } \\
\text { discapacidad, limitación }\end{array}$ & $\begin{array}{l}\text { Discriminación } \\
\text { Predicción } \\
\text { Evaluación }\end{array}$ & 70 \\
\hline $\begin{array}{l}\text { The "How are you" } \\
\text { questionnaire: HAY }\end{array}$ & $\begin{array}{l}\text { Validación } \\
\text { Confiabilidad }\end{array}$ & $\begin{array}{l}\text { Constructo } \\
\text { Criterio }\end{array}$ & $\begin{array}{l}\text { Prueba repetida } \\
\text { Consistencia interna }\end{array}$ & $\begin{array}{l}\text { Funcionalidad físico, } \\
\text { mental, social, discapacidad }\end{array}$ & Discriminación & 71 \\
\hline $\begin{array}{l}\text { The Activities Scale for } \\
\text { Kids Q uestionnaire:ASK }\end{array}$ & $\begin{array}{l}\text { Validación } \\
\text { Confiabilidad }\end{array}$ & Constructo & Prueba repetida & $\begin{array}{l}\text { Funcionalidad físico, } \\
\text { mental, social, dolor }\end{array}$ & Predicción & 72 \\
\hline
\end{tabular}




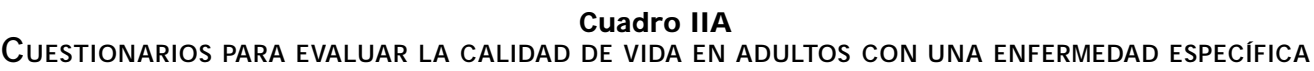

\begin{tabular}{|c|c|c|c|c|c|c|}
\hline Instrumento & Objetivo & Validez & Confiabilidad & Dimensión & Función & Referenci \\
\hline $\begin{array}{l}\text { The Goldman Specific } \\
\text { Activity Scale }\end{array}$ & Validación & $\begin{array}{l}\text { Constructo } \\
\text { Contenido }\end{array}$ & $\begin{array}{l}\text { Prueba repetida } \\
\text { Consistencia interna }\end{array}$ & $\begin{array}{l}\text { Funcionalidad físico, dolor, } \\
\text { limitación }\end{array}$ & $\begin{array}{l}\text { Discriminación } \\
\text { Predicción } \\
\text { Evaluación }\end{array}$ & 73 \\
\hline $\begin{array}{l}\text { The Seattle Q uestionnaire: } \\
\text { SAQ }\end{array}$ & $\begin{array}{l}\text { Validación } \\
\text { Confiabilidad }\end{array}$ & Criterio & $\begin{array}{l}\text { Prueba repetida } \\
\text { Consistencia interna }\end{array}$ & $\begin{array}{l}\text { Funcionalidad físico, social, } \\
\text { emocional, dolor, } \\
\text { limitación }\end{array}$ & $\begin{array}{l}\text { Discriminación } \\
\text { Predicción } \\
\text { Evaluación }\end{array}$ & 74 \\
\hline $\begin{array}{l}\text { The Mild Hypertension Vital } \\
\text { Signs Q uality of Life } \\
\text { Q uestionnaire:VSQ LQ }\end{array}$ & $\begin{array}{l}\text { Validación } \\
\text { Confiabilidad }\end{array}$ & $\begin{array}{l}\text { Constructo } \\
\text { Criterio }\end{array}$ & $\begin{array}{l}\text { Prueba repetida } \\
\text { Consistencia interna }\end{array}$ & Dolor, limitación & $\begin{array}{l}\text { Discriminación } \\
\text { Predicción } \\
\text { Evaluación }\end{array}$ & 75 \\
\hline $\begin{array}{l}\text { The Chronic Venous } \\
\text { Insufficiency Q uestionnaire: } \\
\text { CIVIQ }\end{array}$ & $\begin{array}{l}\text { Validación } \\
\text { Confiabilidad }\end{array}$ & $\begin{array}{l}\text { Constructo } \\
\text { Criterio }\end{array}$ & $\begin{array}{l}\text { Prueba repetida } \\
\text { Consistencia interna }\end{array}$ & $\begin{array}{l}\text { Funcionalidad física, dolor, } \\
\text { limitación, disfunción }\end{array}$ & $\begin{array}{l}\text { Discriminación } \\
\text { Predicción }\end{array}$ & 76 \\
\hline $\begin{array}{l}\text { The Diabetes Q uality of } \\
\text { Life Measure: } D Q O L\end{array}$ & $\begin{array}{l}\text { Validación } \\
\text { Confiabilidad }\end{array}$ & $\begin{array}{l}\text { Constructo } \\
\text { Criterio }\end{array}$ & Consistencia interna & $\begin{array}{l}\text { Satisfacción, metas, } \\
\text { distress, depresión }\end{array}$ & $\begin{array}{l}\text { Discriminación } \\
\text { Predicción }\end{array}$ & 77 \\
\hline $\begin{array}{l}\text { The Diabetes Specific } \\
\text { Q uality of Life Scale for } \\
\text { patients W ith Type I } \\
\text { D iabetes: DSQ O LS }\end{array}$ & $\begin{array}{l}\text { Validación } \\
\text { Confiabilidad }\end{array}$ & $\begin{array}{l}\text { Constructo } \\
\text { Criterio }\end{array}$ & Consistencia interna & $\begin{array}{l}\text { Satisfacción, actividad } \\
\text { física, distress, depresión }\end{array}$ & $\begin{array}{l}\text { Discriminación } \\
\text { Predicción }\end{array}$ & 78 \\
\hline $\begin{array}{l}\text { Q uality of Life } \\
\text { Q uestionnaire for Graves } \\
\text { O phthalmo pathy: GO-Q O L }\end{array}$ & $\begin{array}{l}\text { Validación } \\
\text { Confiabilidad }\end{array}$ & Constructo & Consistencia interna & $\begin{array}{l}\text { Cambios apariencia física, } \\
\text { disminución agudeza } \\
\text { visual, deterioro } \\
\text { psicológico, dolor }\end{array}$ & $\begin{array}{l}\text { Discriminación } \\
\text { Predicción } \\
\text { Evaluación }\end{array}$ & 79 \\
\hline $\begin{array}{l}\text { Q uality of life Parkinson } \\
\text { D isease Q uestionnaire: } \\
\text { PD Q -39 }\end{array}$ & $\begin{array}{l}\text { Validación } \\
\text { Confiabilidad }\end{array}$ & Constructo & Consistencia interna & $\begin{array}{l}\text { Deterioro neurológico, } \\
\text { limitación }\end{array}$ & $\begin{array}{l}\text { Discriminación } \\
\text { Predicción } \\
\text { Evaluación }\end{array}$ & 80 \\
\hline $\begin{array}{l}\text { The Q uality of Life } \\
\text { Q uestionnaire for Multiple } \\
\text { Sclerosis: Q O LQ for MS }\end{array}$ & $\begin{array}{l}\text { Validación } \\
\text { Confiabilidad }\end{array}$ & $\begin{array}{l}\text { Constructo } \\
\text { Criterio }\end{array}$ & Consistencia interna & $\begin{array}{l}\text { Funcionalidad física, } \\
\text { emocional, fatiga }\end{array}$ & $\begin{array}{l}\text { Discriminación } \\
\text { Predicción } \\
\text { Evaluación }\end{array}$ & 81 \\
\hline $\begin{array}{l}\text { The Epilepsy Surgery } \\
\text { Inventory-55: ESI-55 }\end{array}$ & $\begin{array}{l}\text { Validación } \\
\text { Confiabilidad }\end{array}$ & $\begin{array}{l}\text { Constructo } \\
\text { Criterio }\end{array}$ & Consistencia interna & $\begin{array}{l}\text { Deterioro neurológico, } \\
\text { cognición, limitación física }\end{array}$ & $\begin{array}{l}\text { Discriminación } \\
\text { Evaluación }\end{array}$ & 82 \\
\hline $\begin{array}{l}\text { The } Q \text { uality of Life in } \\
\text { Epilepsy: } Q 0 \text { LIE-10 }\end{array}$ & $\begin{array}{l}\text { Validación } \\
\text { Confiabilidad }\end{array}$ & Constructo & $\begin{array}{l}\text { Prueba repetida } \\
\text { Consistencia interna }\end{array}$ & $\begin{array}{l}\text { Funcionalidad físico, social, } \\
\text { mental, cognición, status } \\
\text { poscrisis, relación de } \\
\text { pareja }\end{array}$ & $\begin{array}{l}\text { Discriminación } \\
\text { Evaluación }\end{array}$ & 83 \\
\hline $\begin{array}{l}\text { The Q uality of Life in } \\
\text { Epilepsy: Q O LIE-31 }\end{array}$ & $\begin{array}{l}\text { Validación } \\
\text { Confiabilidad } \\
\text { Traducción al español }\end{array}$ & Constructo & $\begin{array}{l}\text { Prueba repetida } \\
\text { Consistencia interna }\end{array}$ & $\begin{array}{l}\text { Funcionalidad físico, social, } \\
\text { mental, cognición, status } \\
\text { poscrisis, relación de } \\
\text { pareja }\end{array}$ & Discriminación & 84 \\
\hline $\begin{array}{l}\text { The Q uality of Life in } \\
\text { Epilepsy: Q O LIE- } 89\end{array}$ & $\begin{array}{l}\text { Validación } \\
\text { Confiabilidad } \\
\text { Traducción al idioma } \\
\text { de Noruega }\end{array}$ & Constructo & $\begin{array}{l}\text { Prueba repetida } \\
\text { Consistencia interna }\end{array}$ & $\begin{array}{l}\text { Funcionalidad físico, social, } \\
\text { mental, cognición, status } \\
\text { poscrisis, relación de } \\
\text { pareja }\end{array}$ & Discriminación & 85 \\
\hline $\begin{array}{l}\text { The oral health-related } \\
\text { quality of life questionnaire: } \\
\text { OHRQ O L }\end{array}$ & $\begin{array}{l}\text { Validación } \\
\text { Confiabilidad } \\
\text { Traducción al idioma de } \\
\text { N ueva Zelandia, } \\
\text { Alemania y Polonia }\end{array}$ & Constructo & Consistencia interna & $\begin{array}{l}\text { Funcionalidad físico, social, } \\
\text { mental, dolor }\end{array}$ & $\begin{array}{l}\text { Discriminación } \\
\text { Predicción } \\
\text { Evaluación }\end{array}$ & 86 \\
\hline $\begin{array}{l}\text { The Chronic Ear Survey: } \\
\text { CES }\end{array}$ & $\begin{array}{l}\text { Validación } \\
\text { Confiabilidad }\end{array}$ & Criterio & $\begin{array}{l}\text { Prueba repetida } \\
\text { Consistencia interna }\end{array}$ & $\begin{array}{l}\text { Funcionalidad físico, social, } \\
\text { mental, dolor }\end{array}$ & $\begin{array}{l}\text { Discriminación } \\
\text { Predicción } \\
\text { Evaluación }\end{array}$ & 87 \\
\hline $\begin{array}{l}\text { The Chronic Respiratory } \\
\text { Disease Q uestionnaire: CRQ }\end{array}$ & $\begin{array}{l}\text { Validación } \\
\text { Confiabilidad }\end{array}$ & Criterio & $\begin{array}{l}\text { Prueba repetida } \\
\text { Consistencia interna }\end{array}$ & $\begin{array}{l}\text { Funcionalidad físico, social, } \\
\text { mental, dolor, disnea, } \\
\text { fatiga }\end{array}$ & $\begin{array}{l}\text { Discriminación } \\
\text { Predicción } \\
\text { Evaluación }\end{array}$ & 88 \\
\hline
\end{tabular}

\section{(Continúa)}




\section{Cuadro IIA \\ (Continuación)}

\begin{tabular}{|c|c|c|c|c|c|c|}
\hline $\begin{array}{l}\text { The Chronic Respiratory } \\
\text { Disease Q uestionnaire: } \\
\text { CO PD }\end{array}$ & $\begin{array}{l}\text { Validación } \\
\text { Confiabilidad }\end{array}$ & Criterio & $\begin{array}{l}\text { Prueba repetida } \\
\text { Consistencia interna }\end{array}$ & $\begin{array}{l}\text { Funcionalidad física, social, } \\
\text { mental, energía, fatiga }\end{array}$ & $\begin{array}{l}\text { Discriminación } \\
\text { Evaluación }\end{array}$ & 89 \\
\hline $\begin{array}{l}\text { The Measure Yourself } \\
\text { Medical Medical O urtcome } \\
\text { Profile: MYMOP }\end{array}$ & Validación & Constructo & $\begin{array}{l}\text { Prueba repetida } \\
\text { Consistencia interna }\end{array}$ & $\begin{array}{l}\text { Funcionalidad físico, social, } \\
\text { emocional }\end{array}$ & $\begin{array}{l}\text { Discriminación } \\
\text { Predicción }\end{array}$ & 58 \\
\hline $\begin{array}{l}\text { The Marks Asthma Q uality } \\
\text { of Life Q uestionnaire: } \\
\text { AQ LQ }\end{array}$ & $\begin{array}{l}\text { Validación } \\
\text { Confiabilidad }\end{array}$ & Constructo & $\begin{array}{l}\text { Prueba repetida } \\
\text { Consistencia interna }\end{array}$ & $\begin{array}{l}\text { Funcionalidad físico, social, } \\
\text { emocional, síntomas, } \\
\text { dependencia de } \\
\text { tratamiento }\end{array}$ & $\begin{array}{l}\text { Discriminación } \\
\text { Predicción }\end{array}$ & 90 \\
\hline $\begin{array}{l}\text { The Inflammatory Bowel } \\
\text { D isease Q uestionnaire: } \\
\text { IBDQ }\end{array}$ & $\begin{array}{l}\text { Validación } \\
\text { Confiabilidad }\end{array}$ & Constructo & $\begin{array}{l}\text { Prueba repetida } \\
\text { Consistencia interna }\end{array}$ & $\begin{array}{l}\text { Funcionalidad físico, social, } \\
\text { emocional, función } \\
\text { interstinal y sistémica }\end{array}$ & Predicción & 91 \\
\hline $\begin{array}{l}\text { The Dermatology Life } \\
\text { Q uality Index }\end{array}$ & Validación & Constructo & $\begin{array}{l}\text { Prueba repetida } \\
\text { Consistencia interna }\end{array}$ & $\begin{array}{l}\text { Funcionalidad física, social, } \\
\text { emocional }\end{array}$ & Predicción & 92 \\
\hline $\begin{array}{l}\text { The Arthritis Impact } \\
\text { Measurement Scales:AIMS }\end{array}$ & $\begin{array}{l}\text { Validación } \\
\text { Confiabilidad }\end{array}$ & Constructo & Consistencia interna & $\begin{array}{l}\text { Funcionalidad física, social, } \\
\text { emocional, dolor, nivel } \\
\text { actividad }\end{array}$ & $\begin{array}{l}\text { Discriminación } \\
\text { Predicción } \\
\text { Evaluación }\end{array}$ & 93 \\
\hline $\begin{array}{l}\text { The Health Assessment } \\
\text { Q uestionnaire D isability } \\
\text { Index: Spanish HAD-DI }\end{array}$ & $\begin{array}{l}\text { Validación } \\
\text { Confiabilidad } \\
\text { Traducción al español }\end{array}$ & Constructo & $\begin{array}{l}\text { Prueba repetida } \\
\text { Consistencia interna }\end{array}$ & $\begin{array}{l}\text { Funcionalidad física, social, } \\
\text { emocional, dolor, nivel } \\
\text { actividad }\end{array}$ & $\begin{array}{l}\text { Discriminación } \\
\text { Predicción } \\
\text { Evaluación }\end{array}$ & 17 \\
\hline $\begin{array}{l}\text { The Arthritis Impact } \\
\text { Measurements Scales: } \\
\text { Spanish AIMS }\end{array}$ & $\begin{array}{l}\text { Validación } \\
\text { Confiabilidad } \\
\text { Traducción al español }\end{array}$ & Constructo & $\begin{array}{l}\text { Prueba repetida } \\
\text { Consistencia interna }\end{array}$ & $\begin{array}{l}\text { Funcionalidad física, social, } \\
\text { emocional dolor, nivel } \\
\text { actividad }\end{array}$ & $\begin{array}{l}\text { Discriminación } \\
\text { Predicción } \\
\text { Evaluación }\end{array}$ & 19 \\
\hline $\begin{array}{l}\text { The Functional Disability } \\
\text { Index of the Health } \\
\text { Assessment Q uestionnaire: } \\
\text { HAQ FDI }\end{array}$ & $\begin{array}{l}\text { Validación } \\
\text { Confiabilidad } \\
\text { Traducción al italiano }\end{array}$ & Constructo & $\begin{array}{l}\text { Prueba repetida } \\
\text { Consistencia interna }\end{array}$ & $\begin{array}{l}\text { Funcionalidad física, social, } \\
\text { emocional, dolor, nivel } \\
\text { actividad }\end{array}$ & $\begin{array}{l}\text { Discriminación } \\
\text { Predicción } \\
\text { Evaluación }\end{array}$ & 94 \\
\hline $\begin{array}{l}\text { The Shoulder Disability } \\
\text { Q uestionnaire: SDQ }\end{array}$ & $\begin{array}{l}\text { Validación } \\
\text { Confiabilidad }\end{array}$ & Constructo & $\begin{array}{l}\text { Prueba repetida } \\
\text { Consistencia interna }\end{array}$ & $\begin{array}{l}\text { Funcionalidad físico, } \\
\text { emocional, social }\end{array}$ & $\begin{array}{l}\text { Discriminación } \\
\text { Evaluación }\end{array}$ & 95 \\
\hline $\begin{array}{l}\text { The Prostate Cancer } \\
\text { Specific Q uality of Life } \\
\text { Instrument: PRO SQ O LI }\end{array}$ & $\begin{array}{l}\text { Validación } \\
\text { Confiabilidad }\end{array}$ & $\begin{array}{l}\text { Constructo } \\
\text { Criterio }\end{array}$ & $\begin{array}{l}\text { Prueba repetida } \\
\text { Consistencia interna }\end{array}$ & $\begin{array}{l}\text { Funcionalidad físico, social, } \\
\text { emocional, fatiga, } \\
\text { limitación }\end{array}$ & $\begin{array}{l}\text { Discriminación } \\
\text { Predicción } \\
\text { Evaluación }\end{array}$ & 96 \\
\hline $\begin{array}{l}\text { The Schwartz Cancer } \\
\text { Fatigue Scale: SC FS }\end{array}$ & $\begin{array}{l}\text { Validación } \\
\text { Confiabilidad }\end{array}$ & Constructo & $\begin{array}{l}\text { Prueba repetida } \\
\text { Consistencia interna }\end{array}$ & $\begin{array}{l}\text { Funcionalidad físico, social, } \\
\text { vitalidad, fatiga }\end{array}$ & Discriminación & 97 \\
\hline $\begin{array}{l}\text { The Fatigue Severity Scale: } \\
\text { FSS }\end{array}$ & $\begin{array}{l}\text { Validación } \\
\text { Confiabilidad }\end{array}$ & Constructo & $\begin{array}{l}\text { Prueba repetida } \\
\text { Consistencia interna }\end{array}$ & $\begin{array}{l}\text { Funcionalidad físico, social, } \\
\text { vitalidad, fatiga }\end{array}$ & Discriminación & 98 \\
\hline $\begin{array}{l}\text { The Medical O utcomes } \\
\text { Study (MO S) Short form } \\
\text { Health Survey: MO S-HIV }\end{array}$ & $\begin{array}{l}\text { Validación } \\
\text { Confiabilidad }\end{array}$ & $\begin{array}{l}\text { Constructo } \\
\text { Criterio }\end{array}$ & Consistencia interna & $\begin{array}{l}\text { Funcionalidad físico, social, } \\
\text { mental, cognitivo, síntomas } \\
\text { fiebre, fatiga, energía, } \\
\text { dolor, distress }\end{array}$ & Discriminación & 99 \\
\hline $\begin{array}{l}\text { The medical O utcomes } \\
\text { Study HIV Health Survey: } \\
\text { MO S-HIV }\end{array}$ & $\begin{array}{l}\text { Validación } \\
\text { Confiabilidad }\end{array}$ & Constructo & Consistencia interna & $\begin{array}{l}\text { Funcionalidad salud } \\
\text { general, física, social, } \\
\text { mental, cognición, energía, } \\
\text { fatiga }\end{array}$ & Predicción & 100 \\
\hline $\begin{array}{l}\text { The self report HIV-Specific } \\
\text { Q uality of Life: HO PES }\end{array}$ & $\begin{array}{l}\text { Validación } \\
\text { Confiabilidad }\end{array}$ & Constructo & Consistencia interna & $\begin{array}{l}\text { Funcionalidad físico, social, } \\
\text { mental, cognición }\end{array}$ & Discriminación & 101 \\
\hline $\begin{array}{l}\text { The Medical O utcomes } \\
\text { Study Health Survey: } \\
\text { Spanish MO S-HIV }\end{array}$ & $\begin{array}{l}\text { Validación } \\
\text { Confiabilidad } \\
\text { Traducción al español }\end{array}$ & $\begin{array}{l}\text { Constructo } \\
\text { Criterio }\end{array}$ & $\begin{array}{l}\text { Prueba repetida } \\
\text { Consistencia interna }\end{array}$ & $\begin{array}{l}\text { Funcionalidad físico, social, } \\
\text { mental, cognitivo, síntomas } \\
\text { fiebre, fatiga, energía }\end{array}$ & Discriminación & 102 \\
\hline $\begin{array}{l}\text { The Multidimensional } \\
\text { Q uality of Life } \\
\text { Q uestionnaire for HIV: } \\
\text { MQ O L-HIV }\end{array}$ & $\begin{array}{l}\text { Validación } \\
\text { Confiabilidad } \\
\text { Traducción al español }\end{array}$ & $\begin{array}{l}\text { Constructo } \\
\text { Criterio }\end{array}$ & $\begin{array}{l}\text { Prueba repetida } \\
\text { Consistencia interna }\end{array}$ & $\begin{array}{l}\text { Funcionalidad físico, social, } \\
\text { emocional, espiritual, } \\
\text { apoyo social }\end{array}$ & Discriminación & 102 \\
\hline $\begin{array}{l}\text { The HIV-AIDS-Targeted } \\
\text { Q uality of Life: HAT-Q oL }\end{array}$ & $\begin{array}{l}\text { Validación } \\
\text { Confiabilidad }\end{array}$ & Constructo & Consistencia interna & $\begin{array}{l}\text { Funcionalidad físico, } \\
\text { temor control de HIV, } \\
\text { satisfacción }\end{array}$ & Predicción & 103 \\
\hline
\end{tabular}




\section{Cuestionarios para eValuar la Calidad de Vida en Niños Y adolescentes \\ Cuadro IIB \\ CON UNA ENFERMEDAD ESPECÍFICA}

\begin{tabular}{|c|c|c|c|c|c|c|}
\hline Instrumento & Objetivo & Validez & Confiabilidad & Dimensión & Función & Referencia \\
\hline $\begin{array}{l}\text { Calidad de Vida del N iño } \\
\text { con Epilepsia: CAVE }\end{array}$ & $\begin{array}{l}\text { Validación } \\
\text { Confiabilidad }\end{array}$ & Contenido & Prueba repetida & $\begin{array}{l}\text { Conducta, aprendizaje, } \\
\text { autonomía, sociabilidad, } \\
\text { opinión de los padres }\end{array}$ & Discriminación & 104 \\
\hline $\begin{array}{l}\text { The Q uality of Life in } \\
\text { Epilepsy Inventory for } \\
\text { Adolescents: Q O LIE-AD-48 }\end{array}$ & $\begin{array}{l}\text { Validación } \\
\text { Confiabilidad }\end{array}$ & Constructo & $\begin{array}{l}\text { Prueba repetida } \\
\text { Consistencia interna }\end{array}$ & $\begin{array}{l}\text { Funcionalidad memoria, } \\
\text { conducta, actitud, } \\
\text { autoestima, apoyo social }\end{array}$ & Discriminación & 105 \\
\hline $\begin{array}{l}\text { The Q uality of Life in N ewly } \\
\text { Diagnosed Epilepsy } \\
\text { Instrument: N EW Q O L }\end{array}$ & $\begin{array}{l}\text { Validación } \\
\text { Confiabilidad }\end{array}$ & Constructo & $\begin{array}{l}\text { Prueba repetida } \\
\text { Consistencia interna }\end{array}$ & $\begin{array}{l}\text { Actividad social, } \\
\text { psicológicos locus interno, } \\
\text { depresión, ansiedad, } \\
\text { estigma, social, temor, } \\
\text { limitación }\end{array}$ & $\begin{array}{l}\text { Discriminación } \\
\text { Predicción } \\
\text { Evaluación }\end{array}$ & 106 \\
\hline $\begin{array}{l}\text { The Q uality of Life in } \\
\text { Pediatric Epilepsy Scale: } \\
\text { Child Form }\end{array}$ & $\begin{array}{l}\text { Validación } \\
\text { Confiabilidad }\end{array}$ & Constructo & $\begin{array}{l}\text { Prueba repetida } \\
\text { Consistencia interna }\end{array}$ & $\begin{array}{l}\text { Funcionalidad escolar, } \\
\text { cognición, deportes, social, } \\
\text { temor, seguridad, } \\
\text { proyectos a futuro, } \\
\text { autonomía }\end{array}$ & Predicción & 107 \\
\hline $\begin{array}{l}\text { The } Q \text { uality of Life in } \\
\text { Pediatric Epilepsy Scale: } \\
\text { Parents Form }\end{array}$ & $\begin{array}{l}\text { Validación } \\
\text { Confiabilidad }\end{array}$ & Constructo & $\begin{array}{l}\text { Prueba repetida } \\
\text { Consistencia interna }\end{array}$ & $\begin{array}{l}\text { Estigma, cognición, } \\
\text { seguridad, proyectos a } \\
\text { futuro }\end{array}$ & Predicción & 107 \\
\hline $\begin{array}{l}\text { The Q uality of Life in } \\
\text { Childhood Epilepsy } \\
\text { Q uestionnaire: Q O LCE }\end{array}$ & $\begin{array}{l}\text { Validación } \\
\text { Confiabilidad } \\
\text { Descriptivo }\end{array}$ & $\begin{array}{l}\text { Constructo } \\
\text { Criterio }\end{array}$ & Consistencia interna & $\begin{array}{l}\text { Funcionalidad físico, social, } \\
\text { emocional, conducta, } \\
\text { cognición }\end{array}$ & Discriminación & 108 \\
\hline $\begin{array}{l}\text { The O ral Health-related } \\
\text { Q uality of Life } \\
\text { Q uestionnaire: OHRQ OL }\end{array}$ & $\begin{array}{l}\text { Validación } \\
\text { Traducción } \\
\text { Confiabilidad }\end{array}$ & Constructo & Consistencia interna & $\begin{array}{l}\text { Funcionalidad físico, social, } \\
\text { mental, dolor }\end{array}$ & $\begin{array}{l}\text { Discriminación } \\
\text { Predicción } \\
\text { Evaluación }\end{array}$ & 86 \\
\hline $\begin{array}{l}\text { The Sinus Symptoms } \\
\text { Q uestionnaire: SSQ }\end{array}$ & $\begin{array}{l}\text { Validación } \\
\text { Confiabilidad }\end{array}$ & Constructo & Consistencia interna & Limitación & $\begin{array}{l}\text { Discriminación } \\
\text { Predicción } \\
\text { Evaluación }\end{array}$ & 109 \\
\hline $\begin{array}{l}\text { The Canadian Acute } \\
\text { Respiratory Illiness and Flu } \\
\text { Scale: CARIFS }\end{array}$ & $\begin{array}{l}\text { Validación } \\
\text { Confiabilidad }\end{array}$ & Constructo & Prueba repetida & $\begin{array}{l}\text { Funcionalidad físico, } \\
\text { severidad }\end{array}$ & $\begin{array}{l}\text { Discriminación } \\
\text { Predicción }\end{array}$ & 110 \\
\hline $\begin{array}{l}\text { The Pediatric Asthma } \\
\text { Q uality of Life } \\
\text { Q uestionnaire: PAQ LQ }\end{array}$ & $\begin{array}{l}\text { Validación } \\
\text { Confiabilidad }\end{array}$ & Constructo & Consistencia interna & $\begin{array}{l}\text { Funcionalidad físico, } \\
\text { limitación, afecto, ansiedad }\end{array}$ & $\begin{array}{l}\text { Discriminación } \\
\text { Predicción } \\
\text { Evaluación }\end{array}$ & 111 \\
\hline $\begin{array}{l}\text { The Modiffied and Shortend } \\
\text { Version on the Living with } \\
\text { Asthma Q uestionnaire: } \\
\text { ms-LW AQ }\end{array}$ & $\begin{array}{l}\text { Validación } \\
\text { Confiabilidad }\end{array}$ & Constructo & Consistencia interna & $\begin{array}{l}\text { Funcionalidad físico, } \\
\text { limitación, afecto, ansiedad }\end{array}$ & $\begin{array}{l}\text { Discriminación } \\
\text { Predicción } \\
\text { Evaluación }\end{array}$ & 112 \\
\hline $\begin{array}{l}\text { The } C \text { aregiver } Q \text { uality of } \\
\text { Life } Q \text { uestionnaire: } \\
\text { PACQ LQ }\end{array}$ & $\begin{array}{l}\text { Validación } \\
\text { Confiabilidad }\end{array}$ & Constructo & Consistencia interna & $\begin{array}{l}\text { Funcionalidad físico, } \\
\text { limitación, afecto, ansiedad }\end{array}$ & $\begin{array}{l}\text { Discriminación } \\
\text { Predicción } \\
\text { Evaluación }\end{array}$ & 113 \\
\hline $\begin{array}{l}\text { The Juvenile Arthritis } \\
\text { Functional Status Index: } \\
\text { JASI }\end{array}$ & $\begin{array}{l}\text { Validación } \\
\text { Confiabilidad }\end{array}$ & $\begin{array}{l}\text { A parente } \\
\text { Contenido }\end{array}$ & Prueba repetida & $\begin{array}{l}\text { Funcionalidad físico, } \\
\text { mental, social, dolor, } \\
\text { coordinación motora fina } \\
\text { y gruesa }\end{array}$ & Predicción & 114 \\
\hline $\begin{array}{l}\text { The Juvenile Arthritis } \\
\text { Q uality of Life } \\
\text { Q uestionnaire: JAQ Q }\end{array}$ & $\begin{array}{l}\text { Validación } \\
\text { Confiabilidad }\end{array}$ & Constructo & Prueba repetida & $\begin{array}{l}\text { Funcionalidad físico, } \\
\text { mental, social, dolor, } \\
\text { coordinación motora fina } \\
\text { y gruesa }\end{array}$ & Predicción & 115 \\
\hline
\end{tabular}

\section{(Continúa)}




\begin{tabular}{|c|c|c|c|c|c|c|}
\hline \multicolumn{7}{|c|}{$\begin{array}{c}\text { Cuadro IIB } \\
\text { (ConTINUACIón) }\end{array}$} \\
\hline $\begin{array}{l}\text { The Childhood Arthritis } \\
\text { Health Profile: CHAP }\end{array}$ & $\begin{array}{l}\text { Validación } \\
\text { Confiabilidad }\end{array}$ & Constructo & $\begin{array}{l}\text { Prueba repetida } \\
\text { Consistencia interna }\end{array}$ & $\begin{array}{l}\text { Funcionalidad físico, } \\
\text { mental, social, dolor }\end{array}$ & $\begin{array}{l}\text { Discriminación } \\
\text { Predicción }\end{array}$ & 116 \\
\hline $\begin{array}{l}\text { Escala de Actividades del } \\
N \text { iño con Leucemia }\end{array}$ & $\begin{array}{l}\text { Validación } \\
\text { Confiabilidad }\end{array}$ & $\begin{array}{l}\text { Constructo } \\
\text { Contenido }\end{array}$ & Prueba repetida & $\begin{array}{l}\text { Funcionalidad físico, social, } \\
\text { familiar emocional, } \\
\text { competencia académica, } \\
\text { apoyo social }\end{array}$ & Discriminación & 24 \\
\hline $\begin{array}{l}\text { The Pediatric O ncology } \\
\text { Q uality of Life Scale: } \\
\text { PO Q O LS }\end{array}$ & $\begin{array}{l}\text { Validación } \\
\text { Confiabilidad }\end{array}$ & Constructo & $\begin{array}{l}\text { Prueba repetida } \\
\text { Consistencia interna }\end{array}$ & $\begin{array}{l}\text { Funcionalidad físico, social, } \\
\text { emocional, cognitivo, } \\
\text { competencia académica, } \\
\text { apoyo social }\end{array}$ & $\begin{array}{l}\text { Discriminación } \\
\text { Predicción }\end{array}$ & 117 \\
\hline $\begin{array}{l}\text { The Pediatric Cancer } \\
\text { Q uality of Life Inventory: } \\
\text { PCQ L }\end{array}$ & $\begin{array}{l}\text { Validación } \\
\text { Confiabilidad }\end{array}$ & Constructo & $\begin{array}{l}\text { Prueba repetida } \\
\text { Consistencia interna }\end{array}$ & $\begin{array}{l}\text { Funcionalidad físico, social, } \\
\text { emocional, cognitivo, } \\
\text { competencia académica, } \\
\text { apoyo social }\end{array}$ & $\begin{array}{l}\text { Discriminación } \\
\text { Predicción }\end{array}$ & 118 \\
\hline $\begin{array}{l}\text { The Pediatric Cancer } \\
\text { Q uality of Life Inventory: } \\
\text { PC Q L-32 }\end{array}$ & $\begin{array}{l}\text { Validación } \\
\text { Confiabilidad }\end{array}$ & Constructo & $\begin{array}{l}\text { Prueba repetida } \\
\text { Consistencia interna }\end{array}$ & $\begin{array}{l}\text { Funcionalidad físico, social, } \\
\text { emocional, cognitivo, } \\
\text { competencia académica, } \\
\text { apoyo social }\end{array}$ & $\begin{array}{l}\text { Discriminación } \\
\text { Predicción }\end{array}$ & 119 \\
\hline
\end{tabular}

dad de vida. En la columna de validación se incluyeron cuatro tipos de validez: aparente, de contenido, de criterio y de constructo. En la columna de confiabilidad, se consignan cuando se evaluó la consistencia del instrumento con base en una prueba repetida (Test-retest), confiabilidad de la mitad de la muestra y a la consistencia interna. El contenido de los instrumentos se refiere a las dimensiones reportadas en la publicación; entre las más importantes se incluyen actividad física, movilidad, autocuidado, comunicación, interacción social, vitalidad y energía, dolor, actividades recreativas y escolares, así como problemas emocionales y mentales. Finalmente, se reporta la función del instrumento en relación con su capacidad de discriminación entre grupos de pacientes, su finalidad evaluativa, que sirve para detectar cambios a través del tiempo, y la capacidad predictiva del instrumento en relación con el desenlace futuro.

\section{Traducción de los instrumentos}

En cuestionarios de diferente idioma se debe hacer una traducción simple, en una primera etapa y, posteriormente, se traduce nuevamente al idioma original (back-translation) para que finalmente se evalúe el cuestionario en la versión traducida al idioma en que se requiere usar (pre-testing). Este proceso permite la aplicación de un cuestionario que sea confiable y comprensible, y que toma en cuenta los aspectos culturales. ${ }^{14}$ Generalmente la traducción se realiza en varias eta- pas: en la fase 1 , se realiza por investigadores involucrados en el estudio la traducción del documento original en inglés al español; en la fase 2, la versión en español obtiene validez de consenso, validez aparente y validez de contenido, después de que cada uno de los ítems es sometido a revisión por profesionales con experiencia en la atención a pacientes con la enfermedad en estudio; en la fase 3, la versión en español se entrega a un traductor oficial, no relacionado con el equipo del trabajo, para obtener una nueva versión de español a inglés; en la fase 4, el documento original y la traducción hecha por un perito oficial (ambos en inglés), son sometidos a comparación para verificar que no existan discrepancias graves entre la versión original y la traducción. ${ }^{15}$

\section{Experiencia en México}

Existen algunas experiencias en México sobre la aplicación de estos instrumentos.

En 1991, en un tercer nivel de atención, con el fin de investigar el apego al tratamiento en pacientes con padecimientos crónicos de pronóstico fatal, se diseñó un cuestionario, útil y de fácil aplicación, para evaluar las tensiones y molestias que origina su enfermedad en su entorno familiar, escolar y social. ${ }^{16}$

En 1993, Cardiel y colaboradores validaron la versión en español del cuestionario: The health assessment questionnaire disability index (Spanish HAQ-DI). Se aplicó a 97 pacientes con diagnóstico de artritis reu- 
matoide, residentes en la Ciudad de México y su área metropolitana; el instrumento demostró ser sensible para detectar cambios así como su utilidad en poblaciones hispanohablantes; la validación y traducción es comparable con la versión original del HAQ-DI. ${ }^{17}$

La evaluación del impacto de la enfermedad y del tratamiento en la calidad de vida de los pacientes es sumamente importante, pero difícil de cuantificar objetivamente. En 1993, en un hospital de tercer nivel de atención se compararon dos índices clínicos de cambio en calidad de vida: el índice de cambio en función máxima de MacKenzie (ICFM) y de cambio de calidad de vida (ICCV) diseñados por el grupo que realizó el estudio; ambos índices se aplicaron a 23 pacientes con diagnósticos de enfermedad crónica, aguda y subaguda, al inicio y al final de la hospitalización. La reproducibilidad interobservador del ICCV fue mayor que la del ICFM. Ambos índices mostraron aceptable sensibilidad al cambio, así como correlación con la opinión del paciente, sus familiares y del médico tratante. Este estudio demostró que es factible obtener mediciones confiables de los cambios de calidad de vida y que ambos índices podrían usarse en ensayos clínicos. ${ }^{18}$

En 1994, Abello-Banfi y colaboradores validaron la versión en español del cuestionario: The arthritis impact measurement scales (Spanish-AIMS), se administró a 97 pacientes con diagnóstico de artritis reumatoide con residencia en la Ciudad de México y área metropolitana; los resultados fueron positivos y sólo queda fomentar su difusión y aplicación. ${ }^{19}$

En 1994 se compararon las condiciones clínicas y la calidad de vida en 25 pacientes diabéticos, con insuficiencia renal terminal, y en programa de diálisis peritoneal continua ambulatoria (DPCA) y diálisis peritoneal continua en hospitalización (DPI); el estudio mostró que la calidad de vida del paciente transferido a DPCA mejoró $52 \%$ respecto a sus condiciones previas en DPI. ${ }^{20}$

En 1995, en un estudio de 100 pacientes con diabetes tipo II no insulino dependientes, se determinó la relación entre el tiempo de evolución de la enfermedad, descontrol metabólico, enfermedades asociadas, complicaciones tardías y la calidad de vida en un primer nivel de atención. El impacto de las complicaciones es enorme; los pacientes presentaron moderada afección en su calidad de vida por la presencia de las complicaciones tardías, seguidas del descontrol metabólico, el tiempo de evolución y las enfermedades asociadas. $^{21}$

En 1996 se validó el cuestionario COOP-Dartmouth para evaluar el estado funcional biopsicosocial en escolares y adolescentes con enfermedad crónica, encontrando que la calidad de vida se puede medir en forma similar a como se hace en adultos. El cuestionario se sometió a validez de consenso, de apariencia de contenido y de constructo. En la calificación global obtenida se observó que la mayoría de los pacientes presentaron un muy buen estado funcional. ${ }^{22}$

En 1996, en un tercer de nivel de atención, se determinó la validez de un instrumento como un indicador de calidad de vida para evaluar el estado funcional de pacientes pediátricos con leucemia. Se incluyeron menores de 2 a 16 años de edad. En forma global, 100\% de los pacientes obtuvo calificación satisfactoria: el nivel de desempeño diario de actividades de los pacientes fue adecuado, el área escolar fue la más afectada, no se relacionó con la etapa de la enfermedad ni con la fase del tratamiento. El instrumento mostró buena consistencia e identificó adecuadamente el nivel de desempeño diario de los pacientes pediátricos con leucemia. ${ }^{23}$

En 1998, se evaluó el efecto de una intervención educativa sobre la calidad de vida en el paciente hipertenso; se evaluaron la intervención educativa y la repercusión del estilo de vida en el control de la enfermedad; el estudio mostró que la intervención educativa es efectiva para modificar la calidad de vida del paciente hipertenso. ${ }^{24}$

En 1999, se realizó otro estudio para evaluar la calidad de vida de pacientes con enfermedades crónicas (asma, diabetes mellitus tipo I, leucemia y VIH/ SIDA), mediante la aplicación de instrumentos genéricos y específicos. La encuesta específica mostró puntuación correspondiente a una buena calidad de vida en pacientes asmáticos y diabéticos; sin embargo, en las encuestas genéricas los pacientes con diabetes controlada obtuvieron mejor puntuación que los pacientes con diabetes mellitus tipo I descontrolada. Los pacientes con leucemia mostraron diferencias según la etapa de tratamiento, presentando una menor puntuación aquellos con inducción a la remisión que los que se encontraban en mantenimiento. Los niños con infección por VIH presentaron diferencias en la encuesta de salud general; aquellos con estadio clínico B obtuvieron una mejor puntuación de calidad de vida, que los pacientes con estadio clínico C. Finalmente, la comparación entre los cuatro grupos de pacientes sugiere que calidad de vida está relacionada con el control del padecimiento de base. ${ }^{25}$

En otro estudio se aplicó la Encuesta SF-36 en una población en el sureste de México. La encuesta incluye ocho escalas relacionadas con función física, rol físico, dolor corporal, salud general, vitalidad, función social, rol emocional y salud mental. Los resultados obtenidos mostraron que en la población enferma la es- 
cala con más bajo promedio fue la de salud general, y la más alta la del rol físico. En la población sana la escala con más alto promedio fue función física y la más baja salud general. La comparación de promedios de escalas en ambos grupos mostró diferencias significativas en función física, rol físico, dolor corporal, salud general y vitalidad. Los autores concluyen que la encuesta FS-36 cumple en forma satisfactoria con todos los supuestos de validez y confiabilidad. ${ }^{26}$

\section{Conclusiones}

La medición de la calidad de vida presenta retos metodológicos que se han venido resolviendo, y que han permitido desarrollar instrumentos aplicables a diversas enfermedades y poblaciones. Los problemas metodológicos que enfrenta la medición de calidad de vida son dos; primero, que se trata de medidas consideradas "blandas", y segundo, que no hay un valor de referencia estándar de oro contra el cual se pueda comparar. Las dos pruebas más importantes a las cuales deben someterse estas mediciones son las de validez y consistencia; una vez que cumplan estos atributos podrá aceptarse que las mediciones tengan calidad científica. Es decir, si los instrumentos miden realmente lo que se quiere medir y si esta medición es consistentemente reproducible por el mismo observador o por otros. Actualmente se cuenta con dos tipos de instrumentos: los genéricos, que permiten evaluar grupos y poblaciones con diversos diagnósticos, y los específicos, que son de mayor utilidad para medir el impacto de una enfermedad específica. Los instrumentos para medir la calidad de vida disponibles actualmente son confiables y constituyen una herramienta complementaria para evaluar la respuesta al tratamiento. Estos instrumentos también han sido evaluados en función de su capacidad de discriminación, descripción y predicción de la calidad de vida. Finalmente, hay que reconocer que la mayoría de los instrumentos disponibles han sido desarrollados en el idioma inglés, por lo que su aplicación en países de habla hispana requiere no sólo de métodos de traducción válidos, sino reconocer que son específicos al contexto social, por lo que se debe asegurar que los dominios explorados sean apropiados para la población donde se van a implantar.

\section{Referencias}

1. Gill T, Feinstein AR. A critical appraisal of the quality of life measurements. JAMA 1994;272:619-625.

2. Bombardier C, Tugwell P. Methodologic considerations in functional assessment. J Rheumatol 1987;14(Suppl 15):6-12.
3. Patrick DL, D eyo RA. Generic and disease specific measures in assessing health status and quality of life. Med C are 1989;27:217-232.

4. Cardiel MH. ¿Cómo se evalúa la calidad de vida? En:Temas de medicina interna. Epidemiología clínica.A sociación de Medicina Interna de México, A. C. México, D.F.: Interamericana 1994;2:359-368.

5. Bonomi AE, Patrick DI, Bushnell D M, Martin M.Validation of the United States version of W orld Health 0 rganization quality of life (W HOQ O L) instrument. J Clin Epidemiol 2000;53:1-12.

6. Feinstein AR. Preparing for correlated analysis. En: Feinstein AR. Multivariable analysis. $1^{\text {st }}$ Ed. N ashville, Tennessee:Yale University, 1996: 85-188, 7. G uyatt GH, Fenny HD, Patrick DL. Measuring health-related quality of life. Ann Inter Med 1993;118:622-629.

8. Ferrell $B R, W$ isdom $C H, W$ enzl $C$. Q uality of life as an outcome variable in the management of cancer pain. Cancer 1989;63:2321-2327.

9. Kirshner B, G uyatt G.A methodological framework for assessing health indices. J Chron D is 1981;38:27-36.

10. Cronbach LJ. C oefficient alpha and the internal structure of tests. Psychometrika 1951;16:297-334.

11. Cuevas UML, Reynaga O J, Garduño EJ. Tau b de Kendall para datos agrupados. Señalamiento de discrepancia. Rev Med IMSS 1994;32:299-302. 12. Landis RJ, Koch GG. The measurement of interrater agreement. En: Statistics methods for rates and proportions. $2^{\text {st }}$. Edition. N ew York: John W iley and Sons, 1981:212-236.

13. W are J. Standard for validating health measures: D efinition and content. J Chron D is 1998;40(Suppl):473-480.

14. Guillemin F, Bombardier C, Beaton D. Cross-cultural adaptation of health-related quality of life measures: Literature review and proposed guidelines. J Clin Epidemiol 1993;46:1417-1432.

15. $G$ andek $B, W$ are JE. Methods for validating and norming translations of health status questionnaires:The IQ 0 LA project approach. J Clin Epidemiol 1998:51:953-959.

16. Foncerrada MM. La responsabilidad del médico ante los niños enfermos de padecimientos amenazantes para la vida o para la calidad de ésta y en la etapa terminal de las enfermedades. Rev Med IMSS 1991:29:33-36. 17. C ardiel MH,A bello-Banfi M, Ruiz-Mercado R,Alarcón-Segovia D. How to measure health status in rheumatoid arthritis non-english speaking patients:Validation of spanish version of the health assessment questionnaire disability index (Spanish HAD-DI).C lin Exp Rheumatol 1993;11:117121.

18. G uzmán J, Ponce de León S, Pita RL, C astillo RC , Pérez PI. El cambio en la calidad de vida como indicador del curso clínico de la enfermedad. Comparación de dos índices. Rev Invest C lin 1993;45:439-452.

19.A bello-Banfi M,C ardiel MH, Ruiz-Mercado R,Alarcón-Segovia D. Q uality of life in rheumatoid arthritis. Validation of a spanish version of the arthritis impact measurement scales: spanish AIMS. J Rheumatol 1994;21:1250-1255.

20. Becerril PR, Salmerón CA, Ramírez GE, Belio CF. N uevos criterios de asignación a programas de diálisis peritoneal. Impacto sobre la calidad de vida. Rev Med IMSS 1994:32:165-171.

21. MA, Reyes M MH, Garduño EJ, Fajardo GA, Martínez MC. La calidad de vida en el paciente diabético II y factores asociados. Rev Med IMSS 1995:33:293-298.

22. López GA,Vloís FL,A rias GJ,Alonso VF, Cárdenas N R, Villasís KMA et al.Validación del cuestionario C 0 O P-D artmouth para evaluar estado funcional biopsicosocial en escolares y adolescentes con enfermedad crónica. Bol Med Infant Mex 1996;53:606-615.

23. López GA, Hernández HD, Benítez AH, Villasís KMA, Bermádez RR, Martínez GMA. Un instrumento para medir la calidad de vida por medio del desempeño diario en pacientes pediátricos con leucemia. $G$ ac Med Mex 1996;132:19-28.

24. Barrón AJR, Torreblanca RFL, Sánchez CLI, Martínez BMM. Efecto de una intervención educativa en la calidad de vida del paciente hipertenso. Salud Publica Mex 1998;40:503-509. 
25. Toledo BME, N andy LME, Avila-Figueroa C. Evaluación de calidad de vida en pacientes pediátricos con enfermedades crónicas mediante el empleo de cuestionarios específicos y genéricos como instrumentos de medición. Tesis de pediatría médica. Universidad $\mathrm{N}$ acional Autónoma de México. Hospital Infantil de México, 1999.

26. Zúñiga AM, Carrillo JGT, Fos PJ, Gandek B, Medina MRM. Evaluación del estado de salud con la encuesta SF-36. Resultados preliminares en México. Salud Publica Mex 1999:41:110-118.

27. Bergner M, Bobbitt RA, Carter W B, Gilson BS. The sickness impact profile: Development and final revision of a health status measure. Med care 1981;19:787-805.

28. Deyo RA. Pitfalls in measuring the health status mexican americans:A comparative validity of the English and Spanish sickness impact profile. Am J Public Health 1984;74:569-573.

29. Badia X,Alonso J. Re-scaling the spanish version of the sickness impact profile:An opportunity for the assessment of cross- cultural equivalence. J Clin Epidemiol 1995;48:949-957.

30. N elson EC, Landgraf JM, Hays RD, W asson JH, Kirk JW. The functional status of patients. H ow can it be measured in physician offices? Med $\mathrm{C}$ are 1990;28:1111-1126.

31. C hambers LW, MacD onald LA, Tugwell P.The McMaster health index questionnaire as measure of quality of life for patients with rheumatoid disease. J Rheumatol 1982;9:780-786.

32. Kazis LE,Anderson JJ, Meenan RF. Effect sizes for interpreting changes in health status. Med Care 1989;27(Suppl):178-189.

33. Fletcher AE, Hunt BM, Bulpitt C). Evaluation of quality of life in clinical trials of cardiovascular disease. J Chron D is 1987;40:557-566.

34. Parkerson GR, Broadhead W E, Chiu-Kit JT.The Duke health profile.A 17- Item measure of health and dysfunction. Med Care 1990;28:10561071.

35. Liang MH, Fossel AH, Larson MG. C omparisons of five health status instruments for orthopedic evaluation. Med Care 1990;28:632-642. 36. Brezinski D, Stone P, Muller J,Tofler G, D avis V, Parker C et al. Prognostic significance of the Karnofsky performance status score in patients with acute myocardial infarction: Comparison with the left ventricular ejection fraction and exercise treadmill test performance. A $\mathrm{m} \mathrm{Heart} \mathrm{J}$ 1991;121:1374-1381.

37. W are JE, Sherbourne CD. The Health related quality of life: $H R Q O L$. Med Care 1992;30:473-483.

38. Lovell DJ.The Health Assessment Q uestionnaire: HAQ . J Rheumatol 1992;30:819-824

39. Bruin AF, Buys M,W itte LP, Diederiks PM. The sickness impact profile: SIP 68, a short generic version. First evaluation of the reliability and reproducibility. J Clin Epidemiol 1994;47:863-871.

40. Czyzewski DI, Mariotto MJ, Bartholomew LK, LeCompte, Sockrider MM. Measurement of quality of well being in a child and adolescent cystic fibrosis population. Med Care 1994;32:965-972.

41. Stewart AL, Hays RD, W are JE. The MO S short-form general health survey: Reliability and validity in a patient population. Med Care 1988:26:732-724

42. Bindman $A B$, Keane $D$, Laurie $N$. Measuring health changes among severely III patients. Med Care 1990;28:1142-1152.

43. Lara MMC, Ponce de León S, De la Fuente R. Desarrollo de un instrumento para medir la calidad de vida de pacientes con cáncer. Salud Mental 1996;19(suppl):30-35.

44. Escalante A, Lichtenstein MJ, Ríos N, Hazuda HP. Measuring chronic rheumatic pain in mexican americans: Cross- cultural adaptation of the McG ill pain questionnaire.J Clin Epidemiol 1996;49:1389-1399.

45. Sigurdardottir V, BrandbergY, Sullivan M. Criterion based validation of the EO RTC Q LQ -36 in avanced melanoma. The CIPS questionnaire and proxy raters. Q ual Life Res 1996;5:375-386.

46. Groenvold M, Klee MC, Sprangers MAG. Validation of the EO RTC Q LQ - C 30 quality of life questionnaire through combined qualitative and quantitative assessment of patient-observer agreement. J Clin Epidemiol 1997;50:441-450.

47. Lalonide L, Clarke AE, Joseph L, Mackenzie T, Grover SA. Comparing the psyhometric properties of preference-based an nonpreference-based health-related quality of life in coronary heart disease. Q ual Life Res 1999;8:399-409.

48. Díaz P, Mercier C, Hachey R, C aron J, Boyer G. An evaluation of psychometric properties of the client's questionnaire of the $W$ isconsin quality of life index canadian version: C aW -Q LI. Q ual Life Res 1999;8:509-514. 49. Badia X, Rosset MM, Herdman M. Feasibility, validity EU RO Q O L 5D. Q ual Life Res 1999:8:41-43.

50. Strand V, Tugwell P, Bombardier C, Maetzel A, C rawford B, D orrier $C$ et al. Function and health-related quality of life: MHAQ . Arthritis Rheum 1999;42:1870-1878.

51. Holmes W C, Shea JA.Two approaches to measuring quality of life in the HIV-AIDS population: HAT-QOL and MOS-HIV. Q ual Life Res 1999;8:515-527.

52. Sherbourne CD, H ays RD, Fleishman JA, Vitiello B, Magurder KM, Bing EG et al. Impact of psychiatric conditions on healt-related quality of life in persons with HIV infection. Am J Psychiatry 2000;157:248-254.

53. Patrick DL, Kinne S, Engelberg RA, Perllman. Functional status and perceived quality of life in adults with and without chronic conditions: PQ oL. J Clin Epidemiol 2000;53:779-785.

54. Bonomi AE, D onald PL, Bushnell DM, Martin M.Validation of the United States version of the world health organization quality of life (W HO Q O L) instrument. J Clin Epidemiol 2000;53:71-112.

55. Kevin W S, Smith NE, Assmann A, Assmann S. Distinguishing between quality of life and health status in quality of life research:A meta analisis. Q ual Life Res 2000;8:447-459.

56.W illiams JI. Reflections on assessing quality of life and the W HO Q 0 L100 U.S. version. J Clin Epidemiol 2000;53:13-17.

57. Bradley CJ, Kroll J, Holmes-Rovner M.The health and activities limitation index in patients with acute myocardial infarction. J Clin Epidemiol 2000;53:555-562.

58. Paterson C, Langan CE, MCKaig GA, Anderson PM, Maclaine GDH, Rose $L B$ et al.Assessing patient outcomes in acute exacerbations of chronic bronchitis:The measure your medical outcome profile (MYMOP), medical outcomes study 6-items gereral health survey (MOS-6A) and Euro Q ol (EQ -5d). Q ual Life Res 2000;9:521-527.

59. Lee W, Chi KN. The standard of reporting of health-related quality of life in clinical cancer trials.J Clin Epidemiol 2000;53:451-458.

60. Lansky LL, List MA, Lansky SB. Toward the development of play performance scale for children: PPSC. Cancer 1985;56:1837-1849.

61. Rivara FP, Thompson RS, Thompson DC, Calonge N . Injuries to children and adolescents: Impact on physical health. Pediatrics 1991;88:783788.

62. Stein RE, Jones JD. Functional Status II(R). Med Care 1990;28:10411055.

63. Len C, Goldenberg J, Bosi FM, Hilario MO E, O liveira LM, Saccchetti S. Crosscultural reliability of the childhood health assessment questionnaire: J Rheumatol 1994;24:2349-2352.

64. Arguedas 0 , Anderson GB, Fasth A. The Costa Rica childhood health assessment questionnaire: CR-CHAQ.J Rheumatol 1997;24:2233-2241.

65. Goycochea MA, Robles GJ, Vilchis GE.The childhood health assessment questionnaire: CHAQ .J Rheumal 1997;24:2242-2245.

66. D uffy CM, D uffy W KN . The childhood health assessment questionnaire: CHAQ . Curr O pin Rehumatol 1997;9:440-447.

67. González CJ, G onzález VM, Lorig K. The health related quality of life: HRQ O L.Arthritis Care Res 1997;10:448-456.

68. Varni JW, Seid M, Rode CH. The pediatric quality of Life inventory: PedsQ L. Med Care 1999;37:126-139.

69. Bukstein DA, MCG rath MM, Buchner DA, Landraf J, Gross TF. Evaluation of a short form for measuring health-related quality of life among pediatric asthma patients. J Allergy and Clin Immunol 2000;105:245-251. 
70. Feldman BM, Granland B, MCC ullough L,W right V. D istinction of quality of life, health related quality of life, and health status in children referred for rheumatologic care.J Rheumatol 2000;27:226-233.

71. Coq EM, Boeke AJP, Bezemer PD, Bruil J, Eijk TM. C linimetric properties of a parent report on their offspring s quality of life. J Clin Epidemiol 2000;53:139-146.

72. Loung N L,W illiams JI,Yoshida KK,W right JG . Measurement properties of the activities scale for kids. I Clin Epidemiol 2000;53:125-137.

73. Goldman L, Hashimoto B, Cook EF. Comparative reproducibility and validity of systems for assessing cardiovascular functional class:Advantages of a new specific activity scale. Circulation 1981;64:1227-1232.

74. Spertus JA, W inder JA, D ewhurst TA, D eyo RA, Fihn SD. Monitoring the quality of life in patients with coronary artery disease. Am J C ardiol 1994;74:1240-1244.

75. Leidy N K, Schmier KJ, Bonomi AE, Legro M, Zyczynski T, Kong BW. Psychometric properties of VSQ LQ in black patients with mild hypertension vital signs quality of life questionaire. J N atl Med Assoc 2000;92:550557.

76. Launois R, Reboul M, Henry B. C onstruction and validation of a quality of life questionnaire in chronic lower limb venous insufficiency (CIVIQ). Q ual Life Res 1996;5:539-554.

77. Parkerson GR, Connis RT, Broadhead W E. D isease-specific versus generic measurement of health-related quality of Life in insulin-dependent diabetic patients. Med Care 1993;31:629-639.

78. Bott U, Muhlhauser, 0 vermann $\mathrm{H}$. The diabetes specific quality of life scale for patients with Type I diabetes: DSQ O LS. Diabetes Care 1998;21:757-769.

79. Terwee CB, G erding FW, D ekker MF, Prummel JP, Pol JP,W iersingaW M. Test-retest reliability of GO-Q O L:A disease-specific quality of life questionnaire for patients with Graves ophthalopathy. J Clin Epidemiol 1999:52:875-884

80. Bushnoll MD, Mona ML. Q uality of life Parkinson's D isease:Translation and validation of the US parkinson disease questionnaire: PD Q -39. Q ual Life Res 1999;8345-350.

81. Vickrey BA, Hays RD, Genovese BJ, Myers LW, Ellison GW. Comparison of a generic to disease targeted health related quality of life measures for multiple sclerosis. J Clin Epidemiol 1997;50:557-569.

82.Vickrey BG, H ays RD, G raber BS, Rausch R, Engel J, Brook RH.A healthrelated quality of life instrument for patients evaluated for epilepsy surgery. Med Care 1992;30:299-319.

83. Cramer JA, Perrine K, D evinsky O, Meador K.A brief questionnaire to screen for quality of life in epilepsy:The Q 0 LIE -10. Epilepsia 1996;37:577582.

84. Torres $X$, Arroyo S, A raya S, Pablo J.The spanish version of the quality of life in epilepsy inventory: Q 0 LIE 31. Epilepsia 1999;40:1299-1305.

85. Stavem K, Bjornaes H, Lossius MI. Reliability and validity of noruegan version of the quality of the epilepsy inventory: Q O LIE 89. Epilepsia 1999:41:91-97.

86. Tapsoba H, D eschamps JP, Leclerca MH. Factor analytic study of two questionnaires measuring oral healt-related quality of life among children and adults in N ew Zealand, Germany and Poland: OHRQ O L. Q ual Life Res 2000;9:559-569.

87. W ang PC, N adol JB, Merchant S. Validation of outcomes survey for adults with chronic suppurative otitis media: CES.Annals of 0 tology, Rhinology and Laringology 2000;109:249-254.

88. W yrwich KW, Tierney W M, W olinsky. Further evidence supporting a semi-based criterion for identifying meaningful intra-individual changes in health-related quality of life. Clin Epidemiol 1999;52:861-873.

89. Mahler. How should health-related quality of life be assessed in patients with: CO PD. Chest 2000;117(Suppl):54-57.

90. Katz PP, Eisner MD, Henke J, Shiboski S, Yelin EH, Blanc PD.The marks asthma quality of life questionnaire: Further validation and examination of responsiveness to change. J Clin Epidemiol 1999;52:667-675.
91. Cheung W Y, Garratt AM, Russell It, W illiams JG . A british version of the inflammatory bowel disease questionnaire development and validation: IBD Q . Gastroenterology 2000;53:207-306.

92. Mallon $\mathrm{E}, \mathrm{N}$ ewton JN , Klassen. The quality of life in acne:A comparison with general medical conditions use generic questionnaires. British $J$ Dermatology 1999;140:672-676.

93. LE, Anderson JJ, Meenan RF. Effect sizes for interpreting changes in health status. Med C are 1989:27(Suppl):178-189.

94. Ranza R, Marchesoni A, Calori G, Bianchi G, Braga M, C anazza S et al. The italian version of the functional disability index of the health assessment questionnaire.A reliable instrument for multicenter studies on rheumatoid arthritis. Clin Exp Rheumatol 1993;11:123-128.

95. Heijden GEMG , Leffers P, Boutre LM. Shoulder disability questionnaire design and responsiveness of functional measure. J Clin Epidemiol 2000:53:29-38.

96. Stockler MR, O soba D, Goodwin P, C orey P, Tannock IF. Responsiveness to change in health related quality of life in randomized clinical trial: A comparison of the prostate cancer specific quality of life instrument: PRO SQ O LI with analogous scales form the EO RTC Q LQ -30 and a trial specific module.J Clin Epidemiol 1998;51:137-145.

97. Schwartz A. Fatigue mediates the effects of excercise of quality of life. Q ual Life Res 1999;8:529-538.

98. Kleinman L, Zodet MW, Hakim Z, Aledort J, Barker C, Chan K et al. Psychometric evaluation of the severity scale for use in chronic hepatitis C fatigue. Q ual Life Res 2000;9:499-508.

99.W achtel T, Pitte J, Mor V, Stein M, Fleishman J, Carpenter CH.Q uality of life in persons with human immunodeficiency virus infection: Measurement by the medical outcomes study instrument. Ann Inter Med 1992;116:129-137.

100. Carretero MA, Burgess AP, Soler P, Soler M, C atalán J. Reliability and validity of an HIV-specific health-related quality-of-life measure for use with injecting drug users. AIDS 1996;10:1699-1705.

101. De Boer JB, Sprangers MAG, Aaronson NK, Lange JMA, Van Dam FSAM. A study of the reliability, validity and responsiveness of the HIV overview of problems evaluation system (HO PES) in assessing the quality of life of patients with AIDS and symptomatic HIV infection. Q ual Life Res 1996;5:339-347

102. Badia X, Podzamczer D, García M, López-Lavid CC, Consiglio ED.A randomized study comparing instruments for measuring health-related quality of life in HIV-infected patients. Spanish MO S-HIV and MQ O L-HIV valid group medical outcomes study HIV health survey.AID S 1999;13:17271735.

103. Holmes CW, Shea JA.Two approaches to measuring quality of life in the HIV/AIDS population: HAT-QOL and MOS-HIV. Q ual Life Res 1999:8:515-527

104. Casas FC. Experiencia con el cuestionario de calidad de vida en el niño con epilepsia: CAVE. Rev N eurol 1997;225:415-421.

105. C ramer JA,W estbrook EL, D evinsky O , PerrineK , G lassman BM, C amfield $C$. D evelopment of quality of life in epilepsy inventory for adolescents:The Q 0 LIE-AD-48. Epilepsia 1999;40:1114-1121.

106. A betz L, Jacoby A, Baker GA, McN ulty P. Patient-based assessments of quality of life in newly diagnosed epilepsy patients: Validation of the N EW Q 0 L. Epilepsia 2000;41:1119-1128.

107.Arunkumar,W yllie, Kotagal P, O ng HT,W illiam F. Parent - and patientand validated content for pediatric epilepsy quality of assessment. Epilepsia 2000;41:1474-1484.

108. Sabaz M, C airns DR, Lawson JA, N heu N, Bleasel AF, Bye AME.Validation of new quality of life measure for children with epilepsy. Epilepsia 2000;41:765-774.

109. G orbatt JM, G ellman EF, Liltenberg B.The development and validation of on instrument to assess acute sinus disease in children. Sinus symptoms questionnaire: SSQ . Q ual Life Res 1999;8:225-233. 
110. Jacobs B,Young N C , Dick PT, IPP MM, D utkowski R, D avies HD. Canadian acute respiratory illness and flu scale (CARIFS): D evelopment of a valid measure for childhood respiratory infections. I Clin Epidemiol 2000;53:793-799.

111. Juniper EF, Guyatt GH, Feeny DH, Ferrier PJ, Griffith LE, Townsend. Measuring quality of life in children with asthma. Q ual Life Res 1996;5:3546.

112. Ried LD, N au D P, G rainger-Rosseau TJ. Evaluation of patient's healthrelated quality of life using a modified and shortened version of the living with asthma questionnaire (ms-LWAQ) and the medical outcomes study, short- form 36 (SF-36). Q ual Life Res 1999;8:491-499.

113. Bukstein DA, MCG rath MM, Buchner DA, Landgraf J, G oss TF. Evaluation of short form for health-related quality of life among pediatric asthma patients. J Allergy C lin Immunol 2000;105(2pt):245-251.

114. W right VF, Longo KF, Law M, Goidsmith $\mathrm{CH}, \mathrm{Crombe} \mathrm{V,} \mathrm{Dent} \mathrm{P.} \mathrm{The}$ juvenile arthritis functional status index: JASI a validation study. J Rehumatol 1996;23:1066-1079.
115. D uffy CM,Arsenault L, D uffy W KN . The Juvenile arthritis quality of Life questionnaire: JAQ Q .J Rheumatol 1997;24:738-746.

116.Tucker LB, D e N addo BA,A betz LN .The child arthritis health profile: CHAP.J Rheumatol 1995;38(Suppl):183-187.

117. Bradlyn A, Ritchey AK, Harris CV, Moore IM, 0 brien RT, Parsons SK et al. Q uality of life research in pediatric oncology. $C$ ancer 1996;78:13331339.

118.Varni JW, Katz ER, Seid M, Q uiggins DJ.The pediatric cancer quality of Life inventory: PCQ L I:Instrument development, descriptive statistics, and cross-informant variance.J Behav Med 1998;21:179-204.

119.Varni JW, Katz ER, Seid M, Q uiggins DJ, Friedman-Bender. The Pediatric $C$ ancer $Q$ uality of Life Inventory-32:PC Q L-32:I. Reliability and Validity. Cancer 1998:82:1184-1196. 